\title{
Asymptotic-Preserving Discrete Schemes for Non-Equilibrium Radiation Diffusion Problem in Spherical and Cylindrical Symmetrical Geometries
}

\author{
Xia Cui ${ }^{1, *}$, Zhi-Jun Shen ${ }^{1}$ and Guang-Wei Yuan ${ }^{1}$ \\ ${ }^{1}$ Laboratory of Computational Physics, Institute of Applied Physics and \\ Computational Mathematics, P.O. Box 8009-26, Beijing 100088, China.
}

Received 12 September 2016; Accepted (in revised version) 1 May 2017

\begin{abstract}
We study the asymptotic-preserving fully discrete schemes for nonequilibrium radiation diffusion problem in spherical and cylindrical symmetric geometry. The research is based on two-temperature models with Larsen's flux-limited diffusion operators. Finite volume spatially discrete schemes are developed to circumvent the singularity at the origin and the polar axis and assure local conservation. Asymmetric second order accurate spatial approximation is utilized instead of the traditional first order one for boundary flux-limiters to consummate the schemes with higher order global consistency errors. The harmonic average approach in spherical geometry is analyzed, and its second order accuracy is demonstrated. By formal analysis, we prove these schemes and their corresponding fully discrete schemes with implicitly balanced and linearly implicit time evolutions have first order asymptoticpreserving properties. By designing associated manufactured solutions and reference solutions, we verify the desired performance of the fully discrete schemes with numerical tests, which illustrates quantitatively they are first order asymptotic-preserving and basically second order accurate, hence competent for simulations of both equilibrium and non-equilibrium radiation diffusion problems.
\end{abstract}

AMS subject classifications: 65M08, 80M35, 65M12

Key words: Spherical symmetrical geometry, cylindrical symmetrical geometry, non-equilibrium radiation diffusion problem, fully discrete schemes, asymptotic-preserving, second order accuracy.

\section{Introduction}

Non-equilibrium radiation diffusion problems often appear in inertial confinement fusion (ICF), astrophysical phenomena, combustion and other research fields. Two-

*Corresponding author. Email addresses: cui_xia@iapcm.ac.cn (X. Cui), shen_zhijun@iapcm.ac.cn (Z. J. Shen), yuan_guangwei@iapcm.ac.cn (G. W. Yuan) 
temperature model is a representative non-equilibrium problem. In case thermal equilibration is much faster than radiation diffusion, the system is called to be in its equilibrium limit, and can be described by a one-temperature model, which is the asymptotic limit of the two-temperature model. When simulating non-equilibrium radiation diffusion problems using numerical method, asymptotic-preserving (AP) discrete schemes are favored since they are able to maintain this asymptotic property and appropriate for both non-equilibrium and equilibrium radiation diffusions.

To demonstrate the notion of AP scheme more vividly, people often start from an original problem with a scaling parameter $\varepsilon \in[0,1]$. In this application, $\varepsilon$ may differ in several orders of magnitude from the non-equilibrium regime $(\varepsilon=1)$ to the equilibrium regime $(\varepsilon=0)$. The smaller $\varepsilon$ is, the closer the system is to the equilibrium limit. A discrete scheme of the original problem is called to be asymptotic-preserving if it converges to a scheme consistent with the limit problem when $\varepsilon$ tends to zero [1]. An AP scheme works uniformly with respect to the parameter $\varepsilon$, hence adapts to both the original problem and its limit problem.

There are extensive publications studying the AP schemes and properties for transport and hydrodynamic problems [1-7]. These methods are also extended to other fields. For instance, for $P_{1}$ equation, some AP finite volume schemes were established on unstructured meshes in [4]. For discrete-velocity kinetic equations, some high order AP DG schemes were developed in [8]. For a non-equilibrium radiative transfer system, the classic Marshak wave equation and a higher order equilibrium diffusion approach were studied in [9]. However, studies on AP schemes for radiation diffusion problems are rarely found, although their importance in radiation multi-physics is pointed out and some researches on schemes considering accuracy and fast solutions have been developed [10-14].

Benefited from the work for the implicitly balanced (IB) and linearly implicit (LI) time integrations with asymptotic analysis and qualitative numerical tests in [15], we developed some AP finite volume schemes for non-equilibrium radiation diffusion problem in plane geometry in [16]. Therein the sensitivity of the global accuracy to the boundary flux-limiter approaches is explored, and second order spatial accuracy (i.e., $\mathcal{O}\left(h^{2}\right)$ spatial accuracy where $h$ is a nominal mesh size and as $h \rightarrow 0$ ) is gained. However, that paper does not involve the curve geometrical problems. In practice, many heat transfer and radiation hydrodynamic problems arise in complicated geometry instruments, which are more appropriately modeled by using curved geometric coordinate systems such as spherical and cylindrical coordinates for simulation convenience [17-22]. For example, in [18], a radiation hydrodynamics (RHD) code was developed in Cartesian, cylindrical, and spherical geometries to simulate ICF involved problems. In [22], an axissymmetric three-temperature non-equilibrium RHD code was developed to simulate intense thermal radiation or high-power laser driven radiative shock hydrodynamics in cylindrical and spherical symmetric geometry. In this paper, motivated by these broad applications, we focus on developing AP fully discrete schemes with high accuracy for 
non-equilibrium radiation diffusion in spherical and cylindrical symmetric geometries.

The research is carried out on two-temperature models with Larsen's flux-limited diffusion operators. In construction of the schemes, we apply finite volume spatial discretizations to deal with the singularity at the origin and the polar axis, and to keep local flux conservation meanwhile; to guarantee a higher order consistency error on the global domain, we adopt asymmetric second order spatially discrete approximation for fluxlimiters on boundary and symmetric schemes at interior points. In which we derive some harmonic averages of the diffusion coefficients in spherical and cylindrical coordinates to agree with the discrete flux continuity condition. The discrete coefficient differs slightly from the classical one but performs better in numerical tests. An interesting thing needs to mention is that the second order accuracy of this harmonic average is not naturally attained near the origin or the polar axis due to the singularity. We show its second order accuracy in the spherical case by Taylor expansion and order estimate. Formal analysis is performed to prove the first order asymptotic-preserving property (A description of the AP order though $\mathcal{O}\left(\varepsilon^{n}\right)$ as $\varepsilon \rightarrow 0$ is briefly reviewed in Section 5.) of the semi-discrete schemes, consequently the AP properties for the corresponding fully discrete schemes with IB and LI temporal discretizations are achieved.

In the studies on linear diffusion and transport problems [23-26] or simpler nonlinear heat conduction problems [27], one can use classical strategy such as Laplace (Fourier) transformation for temporal (spatial) variant, or adopt some special function to obtain exact solution. But for scaled non-equilibrium radiation diffusion problems, it is rather difficult to construct exact solutions due to the strong nonlinearity and the scaling parameter. In this paper, we devise some relevant exact solution problems on curved geometric coordinates with features of scaling parameter and strong nonlinearity, and carry out numerical experiments to verify the expected performance of the schemes. It shows quantitatively the fully discrete schemes with IB time evolution are first order asymptoticpreserving and second order accurate as a whole. The convergence property is not affected by time and space step lengths as the scaling parameter tends to zero, hence the schemes are qualified to both non-equilibrium and equilibrium problems. For schemes with usual harmonic average diffusion coefficient, the similar conclusion holds.

The outline of the paper is as follows. In Section 2, we display the non-equilibrium radiation diffusion problem in spherical symmetric geometry and present several spatially discrete schemes for its scaled problem. Next in Section 3, we analyze their asymptotic property, and prove they are first order asymptotic-preserving. Hence their corresponding fully discrete schemes with IB and LI time discretizations also have the AP property. In Section 4, the ideas are extended to cylindrical symmetric problem. Then numerical tests are implemented in Section 5 to validate the theoretical results. Finally conclusions are given in Section 6, and more details regarding the second order accuracy of harmonic average in spherical geometry and the truncation error of the discrete diffusion operator as well as a numerical example for non-AP schemes are supplied in Appendixes. 


\section{Spatially discrete schemes for spherical symmetric non-equilibrium radiation diffusion}

Consider the following non-equilibrium radiation diffusion problem in one-dimensional spherical symmetric geometry

$$
\begin{aligned}
& \frac{\partial E}{\partial t}-\frac{1}{r^{2}} \frac{\partial}{\partial r}\left(r^{2} D_{R} \frac{\partial E}{\partial r}\right)=c \sigma_{a}(B-E), \\
& C_{v} \frac{\partial T}{\partial t}=-c \sigma_{a}(B-E), \\
& \sigma_{a}=\bar{\sigma}_{a} T^{-3}, \\
& B=a_{R} T^{4},
\end{aligned}
$$

where $E$ is the radiation energy, $T$ is the material temperature, $c$ is the speed of light, $\sigma_{a}$ is the absorption opacity, $C_{v}$ is the material specific heat, $\bar{\sigma}_{a}$ is a parametric constant, and $a_{R}$ is the radiation constant. We employ Larsen's form for a flux-limited diffusion coefficient

$$
D_{R}=\frac{c}{\sqrt{\left(3 \sigma_{a}\right)^{2}+\left(\frac{1}{E} \frac{\partial E}{\partial r}\right)^{2}}}
$$

which retains first-order asymptotic accuracy in the diffusion limit for radiation transport $[15,28]$.

First we nondimensionalize (2.1) via the substitutions similar as in [15]:

$$
r \rightarrow r_{r e f} r, \quad t \rightarrow t_{r e f} t, \quad T \rightarrow T_{r e f} T, \quad E \rightarrow a_{R} T_{r e f}^{4} E,
$$

and

$$
\sigma_{a} \rightarrow \sigma_{a_{r e f}} \sigma_{a}, \quad D_{R} \rightarrow \frac{c}{\sigma_{a_{r e f}}} D_{R}, \quad C_{v} \rightarrow a_{R} T_{r e f}^{3} C_{v},
$$

where the subscript "ref" stands for a dimensional reference value for each quantity, and $\sigma_{a_{r e f}}=\bar{\sigma}_{a} T_{r e f}^{-3}$. Denote

$$
D_{R}(T, E)=\frac{1}{\sqrt{\left(3 \sigma_{a}\right)^{2}+\left(\frac{1}{r_{r e f} \sigma_{a_{r e f}}} \frac{1}{E} \frac{\partial E}{\partial r}\right)^{2}}} .
$$

Hence (2.1) can be transformed into

$$
\begin{aligned}
& \frac{r_{r e f}}{c t_{r e f}} \frac{\partial E}{\partial t}-\frac{1}{r_{r e f} \sigma_{a_{r e f}} r^{2}} \frac{\partial}{\partial r}\left(r^{2} D_{R} \frac{\partial E}{\partial r}\right)=r_{r e f} \sigma_{a_{r e f}} \sigma_{a}\left(T^{4}-E\right), \\
& C_{v} \frac{r_{r e f}}{c t_{r e f}} \frac{\partial T}{\partial t}=-r_{r e f} \sigma_{a_{r e f}} \sigma_{a}\left(T^{4}-E\right) .
\end{aligned}
$$


Now we set $C_{v}=1$. Then we identify two nondimensional parameters as in [15]:

$$
\frac{r_{r e f}}{c t_{r e f}} \sim \frac{1}{r_{r e f} \sigma_{a_{r e f}}} \sim \mathcal{E} \ll 1 .
$$

The first parameter is the ratio of the gradient length scale $\left(r_{r e f}\right)$ to the distance streaming photons travel within the dynamical time scale $\left(c t_{r e f}\right)$. The second is the ratio of the absorption mean free path $\left(\sigma_{a_{r e f}}^{-1}\right)$ to the gradient length scale. Since the domain of interest is collision dominated, both of them are expected to be small as $\varepsilon$, the "small" asymptotic scaling parameter. Therefore, we have the scaled non-equilibrium radiation diffusion problem written as

$$
\begin{aligned}
& \varepsilon^{2} \frac{\partial E}{\partial t}-\varepsilon^{2} \frac{1}{r^{2}} \frac{\partial}{\partial r}\left(r^{2} D_{R} \frac{\partial E}{\partial r}\right)=\sigma_{a}(B-E), \\
& \varepsilon^{2} \frac{\partial T}{\partial t}=-\sigma_{a}(B-E),
\end{aligned}
$$

where

$$
\begin{aligned}
& B=T^{4}, \quad \sigma_{a}=T^{-3}, \\
& D_{R}(T, E, \varepsilon)=\frac{1}{\sqrt{\left(3 \sigma_{a}\right)^{2}+\varepsilon^{2}\left(\frac{1}{E} \frac{\partial E}{\partial r}\right)^{2}}} .
\end{aligned}
$$

Also the following "boundary" condition is feasible to describe the spherical symmetric problem

$$
\left.\frac{\partial E}{\partial r}\right|_{r=0}=0
$$

Thinking of the singularity at the origin, it is unadvisable to discretize the scaled PDEs directly. We start from the integral formula and propose the following semi-discrete finite volume schemes

$$
\begin{aligned}
\varepsilon^{2} r_{i}^{2} \frac{\partial E_{i}}{\partial t} & -\varepsilon^{2} \frac{1}{h}\left[\overline{\left(r^{2} D_{R}\right)}\right. \\
& =r_{i}^{2} \sigma_{a i}\left(B_{i}-E_{i}\right), \\
\varepsilon^{2} \frac{\partial T_{i}}{\partial t} & =-\sigma_{a i}\left(B_{i}-E_{i}\right),
\end{aligned}
$$

where $h$ represents the spatial step length, and the diffusion coefficient is imitated in three ways. The first choice (Choice 1 ) is a harmonic average

$$
{\overline{\left(r^{2} D_{R}\right)_{i+\frac{1}{2}}}}=\frac{2\left(r^{2} D_{R}\right)_{i+1}\left(r^{2} D_{R}\right)_{i}}{\left(r^{2} D_{R}\right)_{i+1}+\left(r^{2} D_{R}\right)_{i}},
$$


with

$$
\left(r^{2} D_{R}\right)_{i}=\frac{r_{i}^{2}}{\sqrt{\left(3 \sigma_{a i}\right)^{2}+\varepsilon^{2} \chi_{i}}}
$$

where at interior points $x_{i}(i=1,2, \cdots, I-1)$, the discrete flux-limiter $\chi_{i} \approx\left(\frac{1}{E} \frac{\partial E}{\partial r}\right)_{i}^{2}$ takes the following three forms:

(a) the geometric average of the forward and backward difference quotients

$$
\chi_{i}=\frac{4}{h^{2}}\left|\frac{\left(E_{i+1}-E_{i}\right)\left(E_{i}-E_{i-1}\right)}{\left(E_{i+1}+E_{i}\right)\left(E_{i}+E_{i-1}\right)}\right|
$$

(b) the weighted algebraic average of the forward and backward difference quotients

$$
\chi_{i}=\frac{4}{h^{2}} \frac{E_{i}^{2}\left(E_{i+1}-E_{i-1}\right)^{2}}{\left(E_{i+1}+E_{i}\right)^{2}\left(E_{i}+E_{i-1}\right)^{2}}
$$

(c) the centered difference quotient

$$
\chi_{i}=\left(\frac{E_{i+1}-E_{i-1}}{2 h E_{i}}\right)^{2} .
$$

At boundary points,

$$
\chi_{0}=\tilde{\chi}_{0}=\left(\frac{1}{E_{0}} \frac{\frac{3}{2} E_{0}-2 E_{1}+\frac{1}{2} E_{2}}{h}\right)^{2}, \quad \chi_{I}=\tilde{\chi}_{I}=\left(\frac{1}{E_{I}} \frac{\frac{3}{2} E_{I}-2 E_{I-1}+\frac{1}{2} E_{I-2}}{h}\right)^{2} .
$$

Remark 2.1. Formulae (2.8)-(2.10) give approximations of the flux-limiter at interior points. For boundary points, such symmetric discrete stencil is not available, hence one needs other treatment. The traditional way is using a two-point difference quotient to approximate $\frac{\partial E}{\partial r}$ (namely, "Bnd1"), i.e.,

$$
\chi_{0}=\tilde{\chi}_{0}=\left(\frac{1}{E_{0}} \frac{E_{0}-E_{1}}{h}\right)^{2}, \quad \chi_{I}=\tilde{\chi}_{I}=\left(\frac{1}{E_{I}} \frac{E_{I}-E_{I-1}}{h}\right)^{2} .
$$

According to Taylor expansion,

$$
\left(\frac{\partial E}{\partial r}\right)_{0}=\frac{E_{1}-E_{0}}{h}+\mathcal{O}(h)
$$

etc., so Bnd1 is only first order accurate. Hence we use an asymmetric three-point difference quotient formulation, and define (2.11) (namely, "Bnd2") instead. Noticing that

$$
\left(\frac{\partial E}{\partial r}\right)_{0}=-\frac{\frac{3}{2} E_{0}-2 E_{1}+\frac{1}{2} E_{2}}{h}+\mathcal{O}\left(h^{2}\right)
$$

etc., Bnd2 has second order truncation error. Later in numerical tests, we will show it indeed assures the second order accuracy, which exhibits a great advantage over Bnd1. 
The second choice (Choice 2) for discrete diffusion coefficient is a familiar way using the traditional harmonic average of discrete pure "physical" flux continuity, i.e.,

$$
{\overline{\left(r^{2} D_{R}\right)}}_{i+\frac{1}{2}}=r_{i+\frac{1}{2}}^{2} \bar{D}_{R i+\frac{1}{2}}
$$

where

$$
\bar{D}_{R i+\frac{1}{2}}=\frac{2 D_{R i+1} D_{R i}}{D_{R i+1}+D_{R i}}, \quad D_{R i}=\frac{1}{\sqrt{\left(3 \sigma_{a i}\right)^{2}+\varepsilon^{2} \chi_{i}}},
$$

and the discrete flux-limiter is defined as (2.8)-(2.10) and (2.11).

Remark 2.2. We'd like to mention that in contrast to Choice 2, Choice 1 is deduced from the continuity condition for the discrete flux taking into account not only the physical feather but also the geometric feather of the spherical coordinate, hence from the view of solving partial differential equation, Choice 1 is also a (if not more) natural numerical discretization. To distinguish Choice 1 and Choice 2, sometimes we refer to them as spherical harmonic average and physical harmonic average respectively.

The third choice (Choice 3) is a direct approach

$$
{\overline{\left(r^{2} D_{R}\right)}}_{i+\frac{1}{2}}=\frac{r_{i+\frac{1}{2}}^{2}}{\sqrt{\left(3 \sigma_{a i+\frac{1}{2}}\right)^{2}+\varepsilon^{2}\left(\frac{1}{E_{i+\frac{1}{2}}} \frac{E_{i+1}-E_{i}}{h}\right)^{2}}},
$$

where

$$
E_{i+\frac{1}{2}}=\frac{1}{2}\left(E_{i+1}+E_{i}\right)
$$

and the scaled absorption opacity is simulated by

$$
\text { (d) } \sigma_{a i}=T_{i}^{-3}, \quad \sigma_{a i+\frac{1}{2}}=\frac{1}{2}\left(\sigma_{a i+1}+\sigma_{a i}\right),
$$

or

$$
\text { (e) } \sigma_{a i+\frac{1}{2}}=\left(\frac{T_{i+1}+T_{i}}{2}\right)^{-3}
$$

To date we have defined a formal spatially discrete scheme with (2.5). In fact, it includes several different schemes due to different treatments for diffusion coefficient and flux-limiter. To distinguish the schemes better, we name them according to their spatial discrete strategies.

For simplicity, we refer to the schemes defined with discrete diffusion coefficient Choice 1 and discrete interior flux-limiter (a)-(c) as Schemes (a)-(c), and those with Choice 
2 ("physical") and interior flux-limiter (a)-(c) as Schemes (ap)-(cp). They can be further classified according to the definition of discrete boundary flux-limiter, e.g., we have Scheme (a-Bnd1) and Scheme (a-Bnd2), etc. Since we mainly consider schemes with Bnd2 in this paper, we often abbreviate the characters "-Bnd2" when no confusion occurs later. As to that with diffusion coefficient Choice 3 and absorption opacity (d) or (e), we just call it Scheme (d) or (e).

Remark 2.3. We know scheme (2.5) is second order accurate in space via Taylor expansion without consideration of boundary. In fact, after dividing by $\varepsilon^{2}$ on both sides, the consistency errors of both equations are $\mathcal{O}\left(h^{2}+\varepsilon^{2} h^{2}\right)$, hence they tend to zero uniformly with respect to $\varepsilon$ as $h$ tends to zero. It is easy to show this with diffusion coefficient Choice 2 or 3 . However for Choice 1, although intuitively valid, it is necessary to be illuminated in some details. For points distributed near the origin of coordinates, we should measure its accuracy with caution since $r$ is similar as the step length $h$ in magnitude. In Appendix A, we will show the spherical harmonic average is indeed a second order approach for generic smooth function. Furthermore we give the truncation error for the discrete diffusion operator in Appendix B. Hence when we consider the boundary problem, for the two points adjacent to the boundary, with Bnd2 and homogeneous Neumann boundary condition at $r=0$, the truncation errors of Choices 1 and 2 are respectively $\mathcal{O}\left(h^{2}+\varepsilon^{2} h^{2}\right)$ (at $i=1)$ and $\mathcal{O}\left(h^{2}+\varepsilon^{2} h^{2}\right)$ or $\mathcal{O}\left(\varepsilon^{2} h+h^{2}\right)($ at $i=I-1)$.

A conservative formula can be gained by adding the two equations in (2.5) together,

$$
r_{i}^{2} \frac{\partial\left(T_{i}+E_{i}\right)}{\partial t}-\frac{1}{h}\left[{\overline{\left(r^{2} D_{R}\right)}}_{i+\frac{1}{2}} \frac{E_{i+1}-E_{i}}{h}-{\overline{\left(r^{2} D_{R}\right)}}_{i-\frac{1}{2}} \frac{E_{i}-E_{i-1}}{h}\right]=0 .
$$

\section{Asymptotic analysis for discrete schemes}

This section aims to analyze the asymptotic property of the finite volume schemes.

Using asymptotic expansions such as

$$
E=E^{(0)}+\varepsilon E^{(1)}+\varepsilon^{2} E^{(2)}+\varepsilon^{3} E^{(3)}+\cdots,
$$

etc. as noted in [15], we have the respective zero-th and first order asymptotic problems of (2.2) expressed as

$$
\begin{aligned}
& r^{2} \frac{\partial\left[T^{(0)}+B^{(0)}\right]}{\partial t}-\frac{\partial}{\partial r}\left[\left(r^{2} D_{R}\right)^{(0)} \frac{\partial B^{(0)}}{\partial r}\right]=0, \\
& E^{(0)}=B^{(0)}=\left[T^{(0)}\right]^{4}, \quad \sigma_{a}^{(0)}=\left[T^{(0)}\right]^{-3}, \quad\left(r^{2} D_{R}\right)^{(0)}=\frac{r^{2}}{3 \sigma_{a}^{(0)}}
\end{aligned}
$$


and

$$
\begin{aligned}
& r^{2} \frac{\partial\left[T^{(1)}+B^{(1)}\right]}{\partial t}-\frac{\partial}{\partial r}\left[\left(r^{2} D_{R}\right)^{(0)} \frac{\partial B^{(1)}}{\partial r}+\left(r^{2} D_{R}\right)^{(1)} \frac{\partial B^{(0)}}{\partial r}\right]=0, \\
& E^{(1)}=B^{(1)}=4\left[T^{(0)}\right]^{3} T^{(1)}, \quad \sigma_{a}^{(1)}=-\frac{3 T^{(1)}}{T^{(0)}} \sigma_{a}^{(0)}, \\
& \left(r^{2} D_{R}\right)^{(1)}=-\frac{\sigma_{a}^{(1)}}{\sigma_{a}^{(0)}}\left(r^{2} D_{R}\right)^{(0)} .
\end{aligned}
$$

We have the following asymptotic property.

Lemma 3.1. Spatially discrete scheme (2.5) is a first order asymptotic-preserving scheme for the two-temperature problem (2.2).

Proof. By a similar analysis procedure as in [16], the conclusion is valid for the scheme with the usual discrete diffusion coefficient Choice 2 . Here we only write the proofs for the scheme with Choices 1 and 3. We will keep their common part and separate the two cases when necessary.

(I) First, by substituting Hilbert expansions to $B_{i}=T_{i}^{4}$ and $\sigma_{a i} T_{i}^{3}=1$, and equating the coefficients of $\varepsilon^{0}$ and $\varepsilon^{1}$ respectively, we easily see

$$
\begin{aligned}
& B_{i}^{(0)}=\left[T_{i}^{(0)}\right]^{4}, \\
& B_{i}^{(1)}=4\left[T_{i}^{(0)}\right]^{3} T_{i}^{(1)}, \\
& \sigma_{a i}^{(0)}=\left[T_{i}^{(0)}\right]^{-3}, \\
& \sigma_{a i}^{(1)}=-\frac{3 T_{i}^{(1)}}{T_{i}^{(0)}} \sigma_{a i}^{(0)} .
\end{aligned}
$$

In the case of discrete diffusion coefficient Choice 3, moreover, for scheme with (d) and (e), we find respectively

$$
\begin{aligned}
\sigma_{a i+\frac{1}{2}}^{(0)} & =\frac{\left[T_{i+1}^{(0)}\right]^{-3}+\left[T_{i}^{(0)}\right]^{-3}}{2}, \\
\sigma_{a i+\frac{1}{2}}^{(1)} & =\frac{1}{2}\left[-\frac{3 T_{i+1}^{(1)}}{T_{i+1}^{(0)}} \sigma_{a i+1}^{(0)}-\frac{3 T_{i}^{(1)}}{T_{i}^{(0)}} \sigma_{a i}^{(0)}\right],
\end{aligned}
$$

and

$$
\begin{aligned}
& \sigma_{a i+\frac{1}{2}}^{(0)}=\left[\frac{T_{i+1}^{(0)}+T_{i}^{(0)}}{2}\right]^{-3}, \\
& \sigma_{a i+\frac{1}{2}}^{(1)}=-3 \sigma_{a i+\frac{1}{2}}^{(0)}\left[\frac{T_{i+1}^{(0)}+T_{i}^{(0)}}{2}\right]^{-1} \frac{T_{i+1}^{(1)}+T_{i}^{(1)}}{2} .
\end{aligned}
$$


(II) Denoting

$$
\varphi_{i}\left(\varepsilon^{0}, \varepsilon^{1}, \varepsilon^{2}, \cdots\right)=\sqrt{\left(3 \sigma_{a i}\right)^{2}+\varepsilon^{2} \chi_{i}}
$$

and using Hilbert expansions and a few operations as in [16], we derive

$$
\begin{aligned}
& \varphi_{i}\left(\varepsilon^{0}, \varepsilon^{1}, \varepsilon^{2}, \cdots\right)=3 \sigma_{a i}^{(0)}+\mathcal{O}(\varepsilon), \\
& \frac{\partial \varphi_{i}}{\partial \varepsilon^{1}}\left(\varepsilon^{0}, \varepsilon^{1}, \varepsilon^{2}, \cdots\right) \approx 3 \sigma_{a i}^{(1)} .
\end{aligned}
$$

Thereby

$$
\varphi_{i}\left(\varepsilon^{0}, \varepsilon^{1}, \varepsilon^{2}, \cdots\right)=3 \sigma_{a i}^{(0)}+\varepsilon 3 \sigma_{a i}^{(1)}+\cdots .
$$

Hence, in the case of discrete diffusion coefficient Choice 1, (2.7) allows

$$
\begin{aligned}
r_{i}^{2} & =\left(r^{2} D_{R}\right)_{i} \varphi_{i}\left(\varepsilon^{0}, \varepsilon^{1}, \varepsilon^{2}, \cdots\right) \\
& =\left[\left(r^{2} D_{R}\right)_{i}^{(0)}+\varepsilon\left(r^{2} D_{R}\right)_{i}^{(1)}+\varepsilon^{2}\left(r^{2} D_{R}\right)_{i}^{(2)}+\cdots\right]\left[3 \sigma_{a i}^{(0)}+\varepsilon 3 \sigma_{a i}^{(1)}+\cdots\right] \\
& =3 \sigma_{a i}^{(0)}\left(r^{2} D_{R}\right)_{i}^{(0)}+\varepsilon\left[3 \sigma_{a i}^{(1)}\left(r^{2} D_{R}\right)_{i}^{(0)}+3 \sigma_{a i}^{(0)}\left(r^{2} D_{R}\right)_{i}^{(1)}\right]+\mathcal{O}\left(\varepsilon^{2}\right) .
\end{aligned}
$$

Equating like powers of $\varepsilon$ yields

$$
\begin{aligned}
& \left(r^{2} D_{R}\right)_{i}^{(0)}=\frac{r_{i}^{2}}{3 \sigma_{a i}^{(0)}}, \\
& \left(r^{2} D_{R}\right)_{i}^{(1)}=-\frac{\sigma_{a i}^{(1)}}{\sigma_{a i}^{(0)}}\left(r^{2} D_{R}\right)_{i}^{(0)} .
\end{aligned}
$$

Noticing that

$$
\frac{1}{a_{0}+\varepsilon a_{1}+\varepsilon^{2} a_{2}+\cdots}=\frac{1}{a_{0}}-\varepsilon \frac{a_{1}}{a_{0}^{2}}+\cdots,
$$

when $r_{i} \neq 0$, we acquire from (2.6) that

$$
\begin{aligned}
& 2={\overline{\left(r^{2} D_{R}\right)_{i+\frac{1}{2}}}}\left[\frac{1}{\left(r^{2} D_{R}\right)_{i+1}}+\frac{1}{\left(r^{2} D_{R}\right)_{i}}\right] \\
& =\left[\left(r^{2} D_{R}\right)_{i+\frac{1}{2}}^{(0)}+\varepsilon\left(r^{2} D_{R}\right)_{i+\frac{1}{2}}^{(1)}+\varepsilon^{2}\left(r^{2} D_{R}\right)_{i+\frac{1}{2}}^{(2)}+\cdots\right] \\
& \left\{\frac{1}{\left(r^{2} D_{R}\right)_{i+1}^{(0)}}-\varepsilon \frac{\left(r^{2} D_{R}\right)_{i+1}^{(1)}}{\left[\left(r^{2} D_{R}\right)_{i+1}^{(0)}\right]^{2}}+\cdots+\frac{1}{\left(r^{2} D_{R}\right)_{i}^{(0)}}-\varepsilon \frac{\left(r^{2} D_{R}\right)_{i}^{(1)}}{\left[\left(r^{2} D_{R}\right)_{i}^{(0)}\right]^{2}}+\cdots\right\} .
\end{aligned}
$$


A slight calculation and similar comparison indicates

$$
\begin{aligned}
& \left(r^{2} D_{R}\right)_{i+\frac{1}{2}}^{(0)}=\frac{2\left(r^{2} D_{R}\right)_{i+1}^{(0)}\left(r^{2} D_{R}\right)_{i}^{(0)}}{\left(r^{2} D_{R}\right)_{i+1}^{(0)}+\left(r^{2} D_{R}\right)_{i}^{(0)}}, \\
& \left(r^{2} D_{R}\right)_{i+\frac{1}{2}}^{(1)} \\
& \quad=-\left(r^{2} D_{R}\right)_{i+\frac{1}{2}}^{(0)}\left[\frac{\sigma_{a i+1}^{(1)}}{\sigma_{a i+1}^{(0)}}\left(r^{2} D_{R}\right)_{i}^{(0)}+\frac{\sigma_{a i}^{(1)}}{\sigma_{a i}^{(0)}}\left(r^{2} D_{R}\right)_{i+1}^{(0)}\right]\left[\left(r^{2} D_{R}\right)_{i+1}^{(0)}+\left(r^{2} D_{R}\right)_{i}^{(0)}\right]^{-1} .
\end{aligned}
$$

When $r_{i}=0$, we have

$$
\left(r^{2} D_{R}\right)_{i}=0,{\overline{\left(r^{2} D_{R}\right)}}_{i+\frac{1}{2}}=0
$$

from (2.6), hence

$$
\left(r^{2} D_{R}\right)_{i}^{(0)}=0,\left(r^{2} D_{R}\right)_{i+\frac{1}{2}}^{(0)}=0,\left(r^{2} D_{R}\right)_{i+\frac{1}{2}}^{(1)}=0,
$$

thus (3.13)-(3.14) satisfies naturally.

Similarly as the deduction of (3.11) and (3.12), in the case of Choice 3, due to (2.17), it emerges

$$
\begin{aligned}
& \left(r^{2} D_{R}\right)_{i+\frac{1}{2}}^{(0)}=\frac{r_{i+\frac{1}{2}}^{2}}{3 \sigma_{a i+\frac{1}{2}}^{(0)}}, \\
& \left(r^{2} D_{R}\right)_{i+\frac{1}{2}}^{(1)}=-\frac{\sigma_{a i+\frac{1}{2}}^{(1)}}{\sigma_{a i+\frac{1}{2}}^{(0)}}\left(r^{2} D_{R}\right)_{i+\frac{1}{2}}^{(0)} .
\end{aligned}
$$

(III) Inserting asymptotic expansions into (2.5), comparing the $\mathcal{O}\left(\varepsilon^{0}\right)$ terms on both sides, and noticing (3.3), we have

$$
E_{i}^{(0)}=B_{i}^{(0)}=\left[T_{i}^{(0)}\right]^{4} .
$$

(IV) A similar manipulation to (2.18) gives

$$
r_{i}^{2} \frac{\partial\left[T_{i}^{(0)}+E_{i}^{(0)}\right]}{\partial t}-\frac{1}{h}\left[\left(r^{2} D_{R}\right)_{i+\frac{1}{2}}^{(0)} \frac{E_{i+1}^{(0)}-E_{i}^{(0)}}{h}-\left(r^{2} D_{R}\right)_{i-\frac{1}{2}}^{(0)} \frac{E_{i}^{(0)}-E_{i-1}^{(0)}}{h}\right]=0 .
$$

We can show (3.18)-(3.19) are second order spatial discrete approximations of (3.1) no matter Choice 1 or Choice 3 is made in (2.5).

(V) Plugging asymptotic expansions to (2.5), comparing the coefficient of $\varepsilon^{1}$, and taking note of (3.18), (3.4) and (3.5), we get

$$
E_{i}^{(1)}=B_{i}^{(1)}=4\left[T_{i}^{(0)}\right]^{3} T_{i}^{(1)} .
$$


(VI) An analogous operation of (2.18) delivers the $\mathcal{O}\left(\varepsilon^{1}\right)$ equation

$$
\begin{gathered}
r_{i}^{2} \frac{\partial\left[T_{i}^{(1)}+E_{i}^{(1)}\right]}{\partial t}-\frac{1}{h}\left[\left(r^{2} D_{R}\right)_{i+\frac{1}{2}}^{(0)} \frac{E_{i+1}^{(1)}-E_{i}^{(1)}}{h}-\left(r^{2} D_{R}\right)_{i-\frac{1}{2}}^{(0)} \frac{E_{i}^{(1)}-E_{i-1}^{(1)}}{h}\right] \\
-\frac{1}{h}\left[\left(r^{2} D_{R}\right)_{i+\frac{1}{2}}^{(1)} \frac{E_{i+1}^{(0)}-E_{i}^{(0)}}{h}-\left(r^{2} D_{R}\right)_{i-\frac{1}{2}}^{(1)} \frac{E_{i}^{(0)}-E_{i-1}^{(0)}}{h}\right]=0 .
\end{gathered}
$$

We know that (3.20)-(3.21) are second order approximations of (3.2).

Hence Lemma 3.1 comes into existence.

Denoting $\tau$ as the temporal step length, and integrating Lemma 3.1 and the studies on temporally discrete schemes in [15], we establish the asymptotic properties for the fully discrete schemes consequently.

Theorem 3.1. The following linearly implicit fully discrete scheme

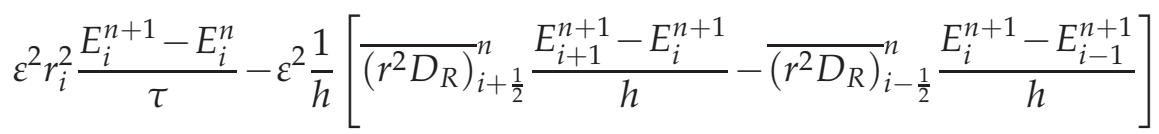

$$
\begin{aligned}
& =r_{i}^{2} \sigma_{a i}^{n}\left[\left(T_{i}^{n}\right)^{4}+4\left(T_{i}^{n}\right)^{3}\left(T_{i}^{n+1}-T_{i}^{n}\right)-E_{i}^{n+1}\right], \\
& \varepsilon^{2} \frac{T_{i}^{n+1}-T_{i}^{n}}{\tau}=-\sigma_{a i}^{n}\left[\left(T_{i}^{n}\right)^{4}+4\left(T_{i}^{n}\right)^{3}\left(T_{i}^{n+1}-T_{i}^{n}\right)-E_{i}^{n+1}\right]
\end{aligned}
$$

is a first order asymptotic-preserving scheme for the two-temperature problem (2.2).

Theorem 3.2. The following implicitly balanced fully discrete scheme

$$
\begin{aligned}
& \varepsilon^{2} r_{i}^{2} \frac{E_{i}^{n+1}-E_{i}^{n}}{\tau}-\varepsilon^{2} \frac{1}{h}\left[\overline{\left(r^{2} D_{R}\right)_{i+\frac{1}{2}}^{n+1}} \frac{E_{i+1}^{n+1}-E_{i}^{n+1}}{h}-{\overline{\left(r^{2} D_{R}\right)_{i-\frac{1}{2}}^{n+1}}}_{\frac{E_{i}^{n+1}-E_{i-1}^{n+1}}{h}}^{h}\right] \\
& =r_{i}^{2} \sigma_{a i}^{n+1}\left[\left(T_{i}^{n+1}\right)^{4}-E_{i}^{n+1}\right], \\
& \varepsilon^{2} \frac{T_{i}^{n+1}-T_{i}^{n}}{\tau}=-\sigma_{a i}^{n+1}\left[\left(T_{i}^{n+1}\right)^{4}-E_{i}^{n+1}\right]
\end{aligned}
$$

is a first order asymptotic-preserving scheme for the two-temperature problem (2.2), and is equilibrium-exact, i.e., it exactly satisfies the equilibrium condition $E=B$ at each time level.

\section{Extension to cylindrical symmetric problem}

The preceding asymptotic analysis can be extended to two-dimensional cylindrical symmetric problems. Consider the following scaled model with (2.4)

$$
\begin{aligned}
& \varepsilon^{2} \frac{\partial E}{\partial t}-\varepsilon^{2} \frac{\partial}{\partial x}\left(D_{R} \frac{\partial E}{\partial x}\right)-\varepsilon^{2} \frac{1}{r} \frac{\partial}{\partial r}\left(r D_{R} \frac{\partial E}{\partial r}\right)=\sigma_{a}(B-E), \\
& \varepsilon^{2} \frac{\partial T}{\partial t}=-\sigma_{a}(B-E)
\end{aligned}
$$


where

$$
\begin{aligned}
& B=T^{4}, \quad \sigma_{a}=T^{-3}, \\
& D_{R}(T, E, \varepsilon)=\frac{1}{\sqrt{\left(3 \sigma_{a}\right)^{2}+\varepsilon^{2}\left[\left(\frac{1}{E} \frac{\partial E}{\partial x}\right)^{2}+\left(\frac{1}{E} \frac{\partial E}{\partial r}\right)^{2}\right]}} .
\end{aligned}
$$

Its zero-th and first order asymptotic equations are respectively

$$
\begin{aligned}
& r \frac{\partial\left[T^{(0)}+B^{(0)}\right]}{\partial t}-r \frac{\partial}{\partial x}\left[D_{R}^{(0)} \frac{\partial B^{(0)}}{\partial x}\right]-\frac{\partial}{\partial r}\left[\left(r D_{R}\right)^{(0)} \frac{\partial B^{(0)}}{\partial r}\right]=0, \\
& E^{(0)}=B^{(0)}=\left[T^{(0)}\right]^{4}, \quad \sigma_{a}^{(0)}=\left[T^{(0)}\right]^{-3}, \quad D_{R}^{(0)}=\frac{1}{3 \sigma_{a}^{(0)}},
\end{aligned}
$$

and

$$
\begin{aligned}
& r \frac{\partial\left[T^{(1)}+B^{(1)}\right]}{\partial t}-r \frac{\partial}{\partial x}\left[D_{R}^{(0)} \frac{\partial B^{(1)}}{\partial x}+D_{R}^{(1)} \frac{\partial B^{(0)}}{\partial x}\right] \\
& -\frac{\partial}{\partial r}\left[\left(r D_{R}\right)^{(0)} \frac{\partial B^{(1)}}{\partial r}+\left(r D_{R}\right)^{(1)} \frac{\partial B^{(0)}}{\partial r}\right]=0, \\
& E^{(1)}=B^{(1)}=4\left[T^{(0)}\right]^{3} T^{(1)}, \quad \sigma_{a}^{(1)}=-\frac{3 T^{(1)}}{T^{(0)}} \sigma_{a}^{(0)}, \quad D_{R}^{(1)}=-\frac{\sigma_{a}^{(1)}}{\sigma_{a}^{(0)}} D_{R}^{(0)} .
\end{aligned}
$$

We apply finite volume discretizations to (4.1)-(4.2). First we denote the harmonic average as follows

$$
D_{R i+\frac{1}{2} j}=\frac{2 D_{R i+1 j} D_{R i j}}{D_{R i+1 j}+D_{R i j}}, \quad{\overline{\left(r D_{R}\right.}}_{i j+\frac{1}{2}}=\frac{2\left(r D_{R}\right)_{i j+1}\left(r D_{R}\right)_{i j}}{\left(r D_{R}\right)_{i j+1}+\left(r D_{R}\right)_{i j}},
$$

with

$$
D_{R i j}=\frac{1}{\sqrt{\left(3 \sigma_{a i j}\right)^{2}+\varepsilon^{2} \chi_{i j}}}, \quad\left(r D_{R}\right)_{i j}=\frac{r_{j}}{\sqrt{\left(3 \sigma_{a i j}\right)^{2}+\varepsilon^{2} \chi_{i j}}},
$$

where $\chi_{i j} \approx\left(\frac{1}{E} \frac{\partial E}{\partial x}\right)_{i j}^{2}+\left(\frac{1}{E} \frac{\partial E}{\partial r}\right)_{i j}^{2}$ has the following three forms:

(a) the geometric average of the forward and backward difference quotients

$$
\chi_{i j}=\frac{4}{h_{1}^{2}}\left|\frac{\left(E_{i+1 j}-E_{i j}\right)\left(E_{i j}-E_{i-1 j}\right)}{\left(E_{i+1 j}+E_{i j}\right)\left(E_{i j}+E_{i-1 j}\right)}\right|+\frac{4}{h_{2}^{2}}\left|\frac{\left(E_{i j+1}-E_{i j}\right)\left(E_{i j}-E_{i j-1}\right)}{\left(E_{i j+1}+E_{i j}\right)\left(E_{i j}+E_{i j-1}\right)}\right|,
$$

(b) the weighted algebraic average of the forward and backward difference quotients

$$
\chi_{i j}=\frac{4}{h_{1}^{2}} \frac{E_{i j}^{2}\left(E_{i+1 j}-E_{i-1 j}\right)^{2}}{\left(E_{i+1 j}+E_{i j}\right)^{2}\left(E_{i j}+E_{i-1 j}\right)^{2}}+\frac{4}{h_{2}^{2}} \frac{E_{i j}\left(E_{i j+1}-E_{i j-1}\right)^{2}}{\left(E_{i j+1}+E_{i j}\right)^{2}\left(E_{i j}+E_{i j-1}\right)^{2}},
$$


(c) the centered difference quotient

$$
\chi_{i j}=\left(\frac{E_{i+1 j}-E_{i-1 j}}{2 h_{1} E_{i j}}\right)^{2}+\left(\frac{E_{i j+1}-E_{i j-1}}{2 h_{2} E_{i j}}\right)^{2} .
$$

Herein $h_{1}$ and $h_{2}$ are respectively the spatial step lengths in the $x$ and $r$ directions.

For boundary points, use the following asymmetric three-point difference quotient approximation of the first order spatial derivatives to guarantee the second order accuracy, e.g., along the $x$ direction,

$$
\left(\frac{\partial E}{\partial x}\right)_{0 j}=-\frac{\frac{3}{2} E_{0 j}-2 E_{1 j}+\frac{1}{2} E_{2 j}}{h_{1}}, \quad\left(\frac{\partial E}{\partial x}\right)_{I j}=\frac{\frac{3}{2} E_{I j}-2 E_{I-1 j}+\frac{1}{2} E_{I-2 j}}{h_{1}},
$$

and corresponding $\chi_{0 j}\left(\tilde{\chi}_{0 j}\right), \chi_{I j}\left(\tilde{\chi}_{I j}\right)$, etc. are defined.

We can design LI and IB fully discrete schemes and derive the asymptotic properties for the semi-discrete and fully discrete schemes similarly, e.g.,

Theorem 4.1. The following linearly implicit fully discrete scheme

$$
\begin{aligned}
& \varepsilon^{2} r_{j} \frac{E_{i j}^{n+1}-E_{i j}^{n}}{\tau}-\varepsilon^{2} r_{j} \frac{1}{h_{1}}\left(D_{R i+\frac{1}{2} j}^{n} \frac{E_{i+1 j}^{n+1}-E_{i j}^{n+1}}{h_{1}}-D_{R i-\frac{1}{2} j}^{n} \frac{E_{i j}^{n+1}-E_{i-1 j}^{n+1}}{h_{1}}\right) \\
& -\varepsilon^{2} \frac{1}{h_{2}}\left[\overline{\left(r D_{R}\right)_{i j+\frac{1}{2}}^{n}} \frac{E_{i j+1}^{n+1}-E_{i j}^{n+1}}{h_{2}}-{\overline{\left(r D_{R}\right)_{i j}}}_{i-\frac{1}{2}}^{n} \frac{E_{i j}^{n+1}-E_{i j-1}^{n+1}}{h_{2}}\right] \\
& \quad=r_{j} \sigma_{a i j}^{n}\left[\left(T_{i j}^{n}\right)^{4}+4\left(T_{i j}^{n}\right)^{3}\left(T_{i j}^{n+1}-T_{i j}^{n}\right)-E_{i j}^{n+1}\right], \\
& \varepsilon^{2} \frac{T_{i j}^{n+1}-T_{i j}^{n}}{\tau}=-\sigma_{a i j}^{n}\left[\left(T_{i j}^{n}\right)^{4}+4\left(T_{i j}^{n}\right)^{3}\left(T_{i j}^{n+1}-T_{i j}^{n}\right)-E_{i j}^{n+1}\right]
\end{aligned}
$$

is a first order asymptotic-preserving scheme for the two-temperature problem (4.1)-(4.2).

Theorem 4.2. The following implicitly balanced fully discrete scheme

$$
\begin{aligned}
& \varepsilon^{2} r_{j} \frac{E_{i j}^{n+1}-E_{i j}^{n}}{\tau}-\varepsilon^{2} r_{j} \frac{1}{h_{1}}\left(D_{R i+\frac{1}{2} j}^{n+1} \frac{E_{i+1 j}^{n+1}-E_{i j}^{n+1}}{h_{1}}-D_{R i-\frac{1}{2} j}^{n+1} \frac{E_{i j}^{n+1}-E_{i-1 j}^{n+1}}{h_{1}}\right) \\
& -\varepsilon^{2} \frac{1}{h_{2}}\left[\overline{\left(r D_{R}\right)_{i j+\frac{1}{2}}^{n+1}} \frac{E_{i j+1}^{n+1}-E_{i j}^{n+1}}{h_{2}}-\overline{\left(r D_{R}\right)_{i j-\frac{1}{2}}^{n+1}} \frac{E_{i j}^{n+1}-E_{i j-1}^{n+1}}{h_{2}}\right] \\
& \quad=r_{j} \sigma_{a i j}^{n+1}\left[\left(T_{i j}^{n+1}\right)^{4}-E_{i j}^{n+1}\right], \\
& \varepsilon^{2} \frac{T_{i j}^{n+1}-T_{i j}^{n}}{\tau}=-\sigma_{a i j}^{n+1}\left[\left(T_{i j}^{n+1}\right)^{4}-E_{i j}^{n+1}\right]
\end{aligned}
$$

is a first order asymptotic-preserving scheme for the two-temperature problem (4.1)-(4.2), and is equilibrium-exact. 
Remark 4.1. It is well known that

$$
\frac{2 u_{j+1} u_{j}}{u_{j+1}+u_{j}}=u_{j+\frac{1}{2}}+\mathcal{O}\left(h^{2}\right)
$$

on the plane. Yet we only have

$$
{\overline{(r u)_{j+\frac{1}{2}}}}=\frac{2(r u)_{j+1}(r u)_{j}}{(r u)_{j+1}+(r u)_{j}}=(r u)_{j+\frac{1}{2}}+\mathcal{O}(h)
$$

near $r=0$ with an analogous estimate procedure in Appendix A. However we see from Appendix B that this does not affect the AP property of the schemes in the cylinder case.

Remark 4.2. Discrete diffusion coefficient (4.5) is based on cylindrical harmonic average (Choice 1). For schemes with a more familiar choice - "physical" harmonic average (Choice 2), e.g., ${\overline{\left(r D_{R}\right)}}_{i j+\frac{1}{2}}=r_{j+\frac{1}{2}} \frac{2 D_{R i j+1} D_{R i j}}{D_{R i j+1}+D_{R i j}}$, the AP property is also valid.

We can name the schemes in (4.11) and (4.12) similarly by combining their spatial and temporal discretizations. For instance, Scheme (a-Bnd1-IB) stands for the scheme with (a-Bnd1) spatial discretization (Choice 1 as discrete diffusion coefficient, formula (a) as discrete interior flux-limiter, first-order discrete boundary flux-limiter) and IB temporal discretization.

Remark 4.3. For the fully discrete schemes in Sections 2 and 4, the consistency error tends to zero with respect to the spatial step length $h$ and temporal step length $\tau$ independently of $\varepsilon$, hence they are asymptotic-preserving.

From Table 3 in Appendix B, we see that for schemes with Choice 1, although the discrete boundary flux-limiters using Bnd1 and Bnd2 themselves have first order and second order accuracy respectively, the truncation error for the corresponding discrete diffusion operator may be very different, even not consistent in some cases. Generally speaking, schemes with Bnd1 are less accurate than Bnd 2 at the points adjacent to the boundary. It is lucky that in the case of our spherical and cylindrical symmetric problem, the schemes are all AP since we have homogeneous Neumann boundary condition (2.4) and then the schemes have perfect consistent error. But if one considers a cylindrical coordinate problem having nonhomogeneous Neumann boundary condition, then the schemes with discrete diffusion coefficient Choice 1 and discrete boundary flux-limiter Bnd1 are not AP, or at least, not well-behaved AP schemes, since at the points near the polar axis, the errors of the schemes are $\mathcal{O}(1)$, which may damage the calculation accuracy to some extent. A numerical example is given in Appendix $\mathrm{C}$ to demonstrate this case.

\section{Numerical tests}

Here we perform numerical experiments to investigate the behavior of the fully discrete schemes and verify the theoretical results. We devise related problems with exact solutions and scaled problem with reference solutions to accomplish the quantitative probe. 
The IB time discretization is employed due to its superiority over the LI method [15]. The numerical results coincide well with our theoretical analysis.

We first check the accuracy of the schemes in spherical geometry. In these tests, we take the mesh ratio $\lambda=\frac{\tau}{h^{2}}=0.1$, and set the convergence error tolerance as $10^{-12}$ without particular remark. Let "error- $\mathrm{E}$ " and "error- $\mathrm{T}$ " respectively represent the discrete maximum norm errors for energy and temperature.

In Sections 2 and 4, we have classified the schemes into several types according to their spatial discrete strategy. Since only fully discrete schemes with IB time evolution are discussed in this section, they inherit the names as there without confusion. For example, here "a-Bnd1" will be an abbreviation of "a-Bnd1-IB", which stands for a scheme with (aBnd1) spatial discretization and IB temporal discretization.

Example 5.1. Consider the model with energy exchange but no scaling parameter

$$
\begin{aligned}
& \frac{\partial E}{\partial t}-\frac{1}{r^{2}} \frac{\partial}{\partial r}\left(r^{2} D_{R} \frac{\partial E}{\partial r}\right)=\sigma_{a}(B-E)+f_{1}(r, t), \\
& \frac{\partial T}{\partial t}=-\sigma_{a}(B-E)+f_{2}(r, t),
\end{aligned}
$$

on spatial domain $(0,1)$. Here $B, \sigma_{a}$ and $D_{R}$ are defined in (2.3) with $\varepsilon=1$. The additional terms are supposed to be

$$
\begin{aligned}
f_{1}(r, t)= & -4\left(r^{4}+1\right) e^{-4 t}-(r+1) e^{-t}+(r+1)\left(r^{4}+1\right) e^{-t} \\
& -\left[9(r+1)^{-6} e^{6 t}+16 r^{6}\left(r^{4}+1\right)^{-2}\right]^{\frac{3}{2}} \\
& \left\{\left[108 r(r+1)^{-1}+180\right] r^{2}(r+1)^{-6} e^{2 t}\right. \\
& \left.+\left[256 r^{4}\left(r^{4}+1\right)^{-1}+128\right] r^{8}\left(r^{4}+1\right)^{-2} e^{-4 t}\right\}, \\
f_{2}(r, t)= & -(r+1)\left(r^{4}+1\right) e^{-4 t} .
\end{aligned}
$$

Its exact solution is $T=(r+1) e^{-t}, E=\left(r^{4}+1\right) e^{-4 t}$.

Numerical errors in the maximum norm for the schemes with different discrete diffusion coefficient choices and boundary flux-limiter approaches at $t=1.5$ are listed in Table 1. Apparently, Schemes (a)-(c) and (ap)-(cp) with Bnd2 are convergent at order 2 as anticipated, and their error data are far less than those with Bnd1. Schemes (d), (e) are also second order convergent.

There are numerous examples exhibiting the ascendancy of Bnd2 over Bnd1 left out here for depiction brevity. Hence we report the subsequent spherical tests only with Bnd2. Also, schemes with coefficient Choice 1 behave slightly better than Choice 2. A possible reason is that when taking into account the discrete flux continuity, by using Choice 1 the diffusion coefficient $r^{2} D_{R}$ is treated as a whole instead of only partly $\left(D_{R}\right)$ as by Choice 2 and hence may be more precise in some cases. Hence we only give the results of the former from now on. 
Table 1: Numerical accuracy of energy and temperature for Example 5.1.

\begin{tabular}{|c|c|c|c|c|c|c|}
\hline scheme & mesh & 50 & 100 & 200 & 400 & order \\
\hline \multirow[t]{2}{*}{ a-Bnd1 } & error-E & $2.11326 \mathrm{e}-4$ & $6.68223 e-5$ & $2.00863 e-5$ & $5.73098 \mathrm{e}-6$ & 1.73485 \\
\hline & error-T & $4.47000 \mathrm{e}-3$ & $1.44350 \mathrm{e}-3$ & $4.37018 \mathrm{e}-4$ & $1.24973 \mathrm{e}-4$ & 1.72019 \\
\hline \multirow[t]{2}{*}{ b-Bnd1 } & error-E & $2.06952 \mathrm{e}-4$ & $6.56592 \mathrm{e}-5$ & $1.97748 \mathrm{e}-5$ & $5.64866 \mathrm{e}-6$ & 1.73175 \\
\hline & error-T & $4.38020 \mathrm{e}-3$ & $1.41862 \mathrm{e}-3$ & $4.30258 \mathrm{e}-4$ & $1.23179 \mathrm{e}-4$ & 1.71739 \\
\hline \multirow{2}{*}{ c-Bnd1 } & error-E & $1.79104 \mathrm{e}-4$ & $5.79613 e-5$ & $1.76547 \mathrm{e}-5$ & $5.07524 \mathrm{e}-6$ & 1.71373 \\
\hline & error-T & $3.80605 \mathrm{e}-3$ & $1.25373 \mathrm{e}-3$ & $3.84251 \mathrm{e}-4$ & $1.10684 \mathrm{e}-4$ & 1.70126 \\
\hline \multirow[t]{2}{*}{ a-Bnd2 } & error-E & $5.35985 \mathrm{e}-5$ & $1.32304 \mathrm{e}-5$ & $3.25552 \mathrm{e}-6$ & $8.02956 \mathrm{e}-7$ & 2.02024 \\
\hline & error-T & $1.16037 \mathrm{e}-3$ & $2.88120 \mathrm{e}-4$ & $7.10071 \mathrm{e}-5$ & $1.75212 \mathrm{e}-5$ & 2.01645 \\
\hline \multirow[t]{2}{*}{ b-Bnd2 } & error-E & $4.83237 \mathrm{e}-5$ & $1.18834 \mathrm{e}-5$ & $2.91090 \mathrm{e}-6$ & $7.15186 \mathrm{e}-7$ & 2.02609 \\
\hline & error-T & $1.04698 \mathrm{e}-3$ & $2.58832 \mathrm{e}-4$ & $6.34917 \mathrm{e}-5$ & $1.56057 \mathrm{e}-5$ & 2.02267 \\
\hline \multirow[t]{2}{*}{ c-Bnd2 } & error-E & $1.61164 \mathrm{e}-5$ & $3.10009 \mathrm{e}-6$ & $5.66505 \mathrm{e}-7$ & $1.01682 \mathrm{e}-7$ & 2.43611 \\
\hline & error-T & $3.50744 \mathrm{e}-4$ & $6.75748 \mathrm{e}-5$ & $1.23508 \mathrm{e}-5$ & $2.21629 \mathrm{e}-6$ & 2.43538 \\
\hline \multirow[t]{2}{*}{ ap-Bnd1 } & error-E & $2.34406 \mathrm{e}-4$ & $7.18977 \mathrm{e}-5$ & $2.12251 \mathrm{e}-5$ & $5.99067 \mathrm{e}-6$ & 1.76338 \\
\hline & error-T & $4.94387 \mathrm{e}-3$ & $1.55243 e-3$ & $4.61791 \mathrm{e}-4$ & $1.30640 \mathrm{e}-4$ & 1.74732 \\
\hline \multirow[t]{2}{*}{ bp-Bnd1 } & error-E & $2.30097 \mathrm{e}-4$ & $7.07407 e-5$ & $2.09142 \mathrm{e}-5$ & $5.90840 \mathrm{e}-6$ & 1.76111 \\
\hline & error-T & $4.85598 \mathrm{e}-3$ & $1.52771 \mathrm{e}-3$ & $4.55046 \mathrm{e}-4$ & $1.28847 \mathrm{e}-4$ & 1.74534 \\
\hline \multirow[t]{2}{*}{ cp-Bnd1 } & error-E & $2.02737 \mathrm{e}-4$ & $6.31066 \mathrm{e}-5$ & $1.88014 \mathrm{e}-5$ & $5.33572 \mathrm{e}-6$ & 1.74926 \\
\hline & error-T & $4.29541 \mathrm{e}-3$ & $1.36440 \mathrm{e}-3$ & $4.09206 \mathrm{e}-4$ & $1.16368 \mathrm{e}-4$ & 1.73534 \\
\hline \multirow[t]{2}{*}{ ap-Bnd2 } & error-E & $6.78096 \mathrm{e}-5$ & $1.67022 \mathrm{e}-5$ & $4.11780 \mathrm{e}-6$ & $1.01814 \mathrm{e}-6$ & 2.01916 \\
\hline & error-T & $1.46549 \mathrm{e}-3$ & $3.63645 \mathrm{e}-4$ & $8.98190 \mathrm{e}-5$ & $2.22180 \mathrm{e}-5$ & 2.01450 \\
\hline \multirow[t]{2}{*}{ bp-Bnd2 } & error-E & $6.26068 \mathrm{e}-5$ & $1.53622 \mathrm{e}-5$ & $3.77383 \mathrm{e}-6$ & $9.30421 \mathrm{e}-7$ & 2.02410 \\
\hline & error-T & $1.35409 \mathrm{e}-3$ & $3.34533 \mathrm{e}-4$ & $8.23187 \mathrm{e}-5$ & $2.03037 \mathrm{e}-5$ & 2.01981 \\
\hline \multirow[t]{2}{*}{ cp-Bnd2 } & error-E & $3.09235 \mathrm{e}-5$ & $6.64287 \mathrm{e}-6$ & $1.43608 \mathrm{e}-6$ & $3.17515 \mathrm{e}-7$ & 2.20191 \\
\hline & error-T & $6.71928 \mathrm{e}-4$ & $1.44820 \mathrm{e}-4$ & $3.13284 \mathrm{e}-5$ & $6.92735 \mathrm{e}-6$ & 2.19995 \\
\hline \multirow[t]{2}{*}{$\mathrm{d}$} & error-E & $4.35889 \mathrm{e}-5$ & $1.08858 \mathrm{e}-5$ & $2.72067 \mathrm{e}-6$ & $6.80130 \mathrm{e}-7$ & 2.00067 \\
\hline & error-T & $9.45407 \mathrm{e}-4$ & $2.37203 e-4$ & $5.93527 \mathrm{e}-5$ & $1.48417 \mathrm{e}-5$ & 1.99774 \\
\hline \multirow[t]{2}{*}{ e } & error-E & $4.56714 \mathrm{e}-5$ & $1.14019 \mathrm{e}-5$ & $2.84938 \mathrm{e}-6$ & $7.12290 \mathrm{e}-7$ & 2.00089 \\
\hline & error-T & $9.90264 \mathrm{e}-4$ & $2.48427 \mathrm{e}-4$ & $6.21594 \mathrm{e}-5$ & $1.55434 \mathrm{e}-5$ & 1.99781 \\
\hline
\end{tabular}

Example 5.2. Now consider a more complex model with both energy exchange and scaling parameter as follows,

$$
\begin{aligned}
& \varepsilon^{2} \frac{\partial E}{\partial t}-\varepsilon^{2} \frac{1}{r^{2}} \frac{\partial}{\partial r}\left(r^{2} D_{R} \frac{\partial E}{\partial r}\right)=\sigma_{a}\left(T^{4}-E\right)+f_{1}(r, t, \varepsilon), \\
& \varepsilon^{2} \frac{\partial T}{\partial t}=-\sigma_{a}\left(T^{4}-E\right)+f_{2}(r, t, \varepsilon),
\end{aligned}
$$

where $B, \sigma_{a}$ and $D_{R}$ are specified with (2.3). The domain of interest is $(0,1)$. The boundary 
and initial conditions respectively read

$$
\begin{aligned}
& \frac{\partial E}{\partial r}(0, t, \varepsilon)=0, E(1, t, \varepsilon)=(1+A)^{2}[\sin (\alpha t+B)+2]^{2} ; \\
& T(r, 0, \varepsilon)=\left(r^{3}+A\right)^{-\frac{1}{2}}(\sin B+2)^{\frac{1}{2}}, \\
& E(r, 0, \varepsilon)=\left(r^{3}+A\right)^{2}(\sin B+2)^{2} .
\end{aligned}
$$

Its exact solution is $T=\left(r^{3}+A\right)^{\frac{1}{2}}[\sin (\alpha t+B)+2]^{\frac{1}{2}}, E=\left(r^{3}+A\right)^{2}[\sin (\alpha t+B)+2]^{2}$. The additional terms can be written accordingly, i.e.,

$$
\begin{aligned}
f_{1}(r, t, \varepsilon)= & 2 \varepsilon^{2} \alpha \cos (\alpha t+B)\left(r^{3}+A\right)^{2}[\sin (\alpha t+B)+2] \\
& -\varepsilon^{2}\left\{9\left(r^{3}+A\right)^{-3}[\sin (\alpha t+B)+2]^{-3}+36 \varepsilon^{2} r^{4}\left(r^{3}+A\right)^{-2}\right\}^{-\frac{3}{2}} \\
& \left\{27 r\left(23 r^{3}+8 A\right)\left(r^{3}+A\right)^{-3}[\sin (\alpha t+B)+2]^{-1}\right. \\
& \left.+432 \varepsilon^{2} r^{5}\left(4 r^{3}+A\right)\left(r^{3}+A\right)^{-2}[\sin (\alpha t+B)+2]^{2}\right\}, \\
f_{2}(r, t, \varepsilon)= & \frac{1}{2} \varepsilon^{2} \alpha \cos (\alpha t+B)\left(r^{3}+A\right)^{\frac{1}{2}}[\sin (\alpha t+B)+2]^{-\frac{1}{2}} .
\end{aligned}
$$

In this test, we set $A=0.5 \varepsilon, B=1+\varepsilon$ and $\alpha=2$, and take the error tolerance as $10^{-10}$. We check the numerical errors at $t=0.1$ with $\varepsilon$ being 1, 0.1, 0.01, 0.001 and 0.0001, and find they all converge at order 2. As examples, Figs. 1-2 respectively present the results for $\varepsilon=1$ and 0.0001 .

Example 5.3. We use reference solutions of the scaled non-equilibrium radiation diffusion problem (2.2) to continue the accuracy tests. Let the spatial domain be $(0,1)$. For its zero-th order asymptotic equation (3.1), we take the following boundary and initial conditions

$$
\begin{aligned}
& \frac{\partial E^{(0)}}{\partial r}(0, t)=0, \quad E^{(0)}(1, t)=\frac{1}{3} e^{-t}+\frac{2}{3} ; \\
& T^{(0)}(r, 0)=1, \quad E^{(0)}(r, 0)=1 .
\end{aligned}
$$

For its first order asymptotic equation (3.2), we take them as

$$
\begin{aligned}
& \frac{\partial E^{(1)}}{\partial r}(0, t)=0, \quad E^{(1)}(1, t)=2 e^{-t}+2 ; \\
& T^{(1)}(r, 0)=1, \quad E^{(1)}(r, 0)=4 .
\end{aligned}
$$

Furthermore, we take $T^{(0)}+\varepsilon T^{(1)}$ and $E^{(0)}+\varepsilon E^{(1)}$ as initial and boundary conditions for the scaled problem (2.2), i.e.,

$$
\begin{aligned}
& \frac{\partial E}{\partial r}(0, t, \varepsilon)=0, \quad E(1, t, \varepsilon)=\frac{1}{3} e^{-t}+\frac{2}{3}+\varepsilon\left(2 e^{-t}+2\right) ; \\
& T(r, 0, \varepsilon)=1+\varepsilon, \quad E(r, 0)=1+4 \varepsilon .
\end{aligned}
$$



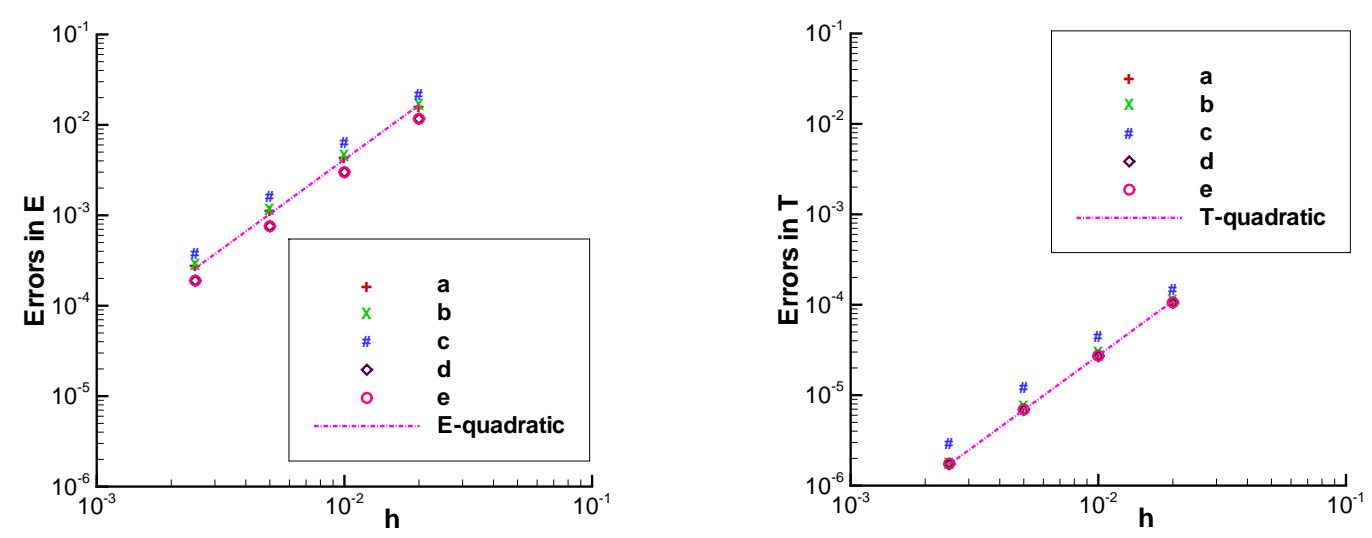

Figure 1: Numerical accuracy of energy (left) and temperature (right) with $\varepsilon=1$ for Example 5.2.
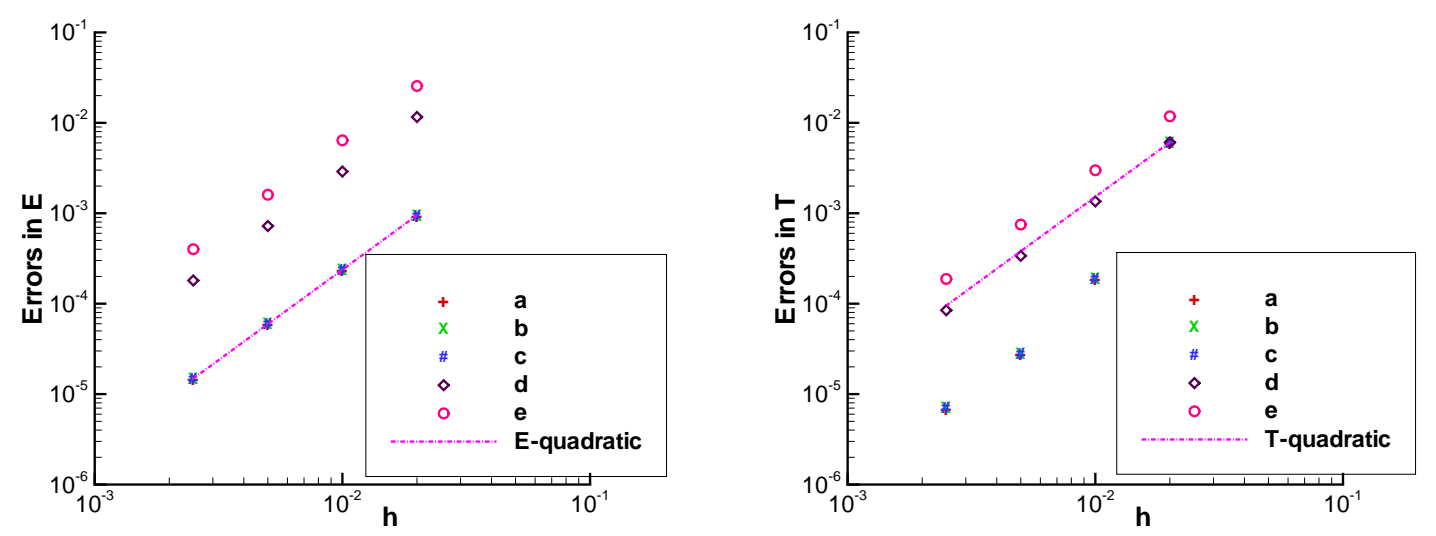

Figure 2: Numerical accuracy of energy (left) and temperature (right) with $\varepsilon=0.0001$ for Example 5.2.

We take the numerical solutions obtained on a refined mesh with $h=1 / 3200$ as reference solutions, and assess the errors between the numerical solutions and the reference solutions with various asymptotic parameters. We observed that the choice of scaling parameter does not affect the second order accuracy of the schemes. As an example, Fig. 3 depicts the results at $t=0.1$ with $\varepsilon=10^{-2}$ and $10^{-12}$. Similar behavior appears with $\varepsilon=1 / 200,1 / 400,1 / 800$, etc.

In the following, we consider the accuracy of the schemes for the cylindrical symmetric problems on a unit square, and take the number of spatial cells as $8 \times 8,16 \times 16,32 \times 32$ and $64 \times 64$. Denote $h=h_{1}=h_{2}$ and the mesh ratio as $\lambda=\frac{\tau}{h^{2}}$. Considering the discrete flux continuity property, only Schemes (a)-(c) are applied in these tests. 

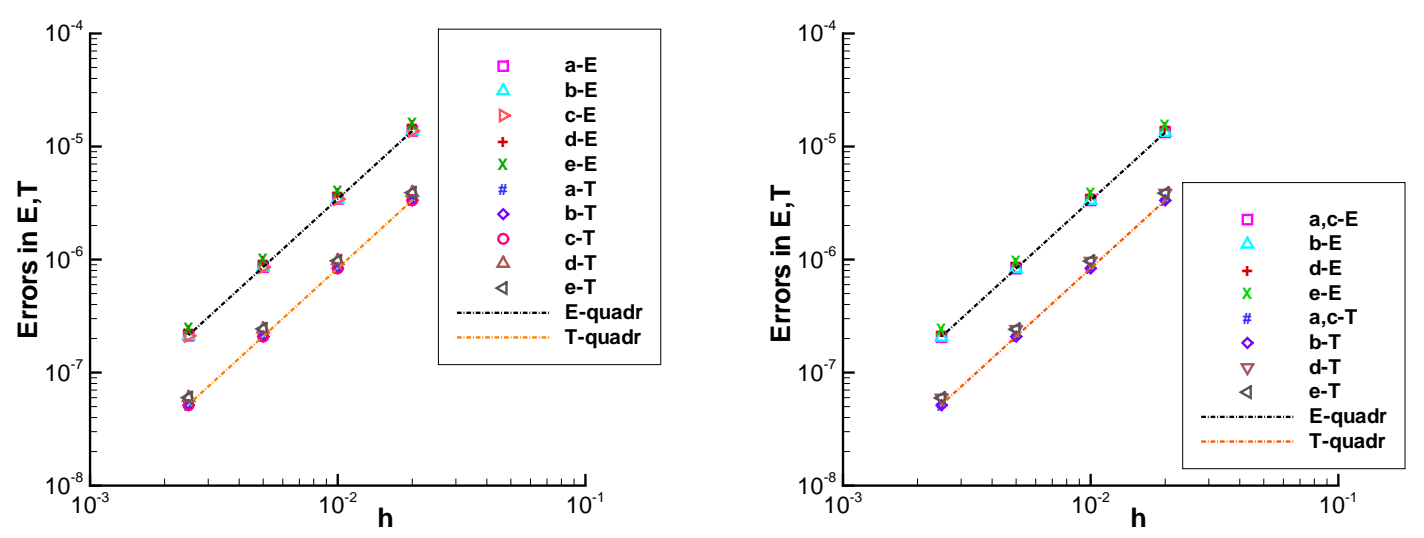

Figure 3: Numerical accuracy of energy and temperature with $\varepsilon=10^{-2}$ (left) and $10^{-12}$ (right) for Example 5.3.

Example 5.4. Consider the model with additional source terms

$$
\begin{aligned}
& \varepsilon^{2} \frac{\partial E}{\partial t}-\varepsilon^{2} \frac{\partial}{\partial x}\left(D_{R} \frac{\partial E}{\partial x}\right)-\varepsilon^{2} \frac{1}{r} \frac{\partial}{\partial r}\left(r D_{R} \frac{\partial E}{\partial r}\right)=\sigma_{a}(B-E)+f_{1}(x, r, t, \varepsilon), \\
& \varepsilon^{2} \frac{\partial T}{\partial t}=-\sigma_{a}(B-E)+f_{2}(x, r, t, \varepsilon),
\end{aligned}
$$

where $B, \sigma_{a}$ and $D_{R}$ are defined in (2.3). The additional terms read

$$
\begin{aligned}
f_{1}(x, r, t, \varepsilon)= & 2 \varepsilon^{2} A\left(x^{2}+r^{2}+C\right)^{2}\left[\sin (A t+B)+\frac{3}{2}\right] \cos (A t+B) \\
& -\varepsilon^{2}\left\{9\left(x^{2}+r^{2}+C\right)^{-3}\left[\sin (A t+B)+\frac{3}{2}\right]^{-3}\right. \\
& \left.+16 \varepsilon^{2}\left(x^{2}+r^{2}\right)\left(x^{2}+r^{2}+C\right)^{-2}\right\}^{-\frac{3}{2}} \\
& \left\{36\left(x^{2}+r^{2}+C\right)^{-3}\left(8 x^{2}+8 r^{2}+3 C\right)\left[\sin (A t+B)+\frac{3}{2}\right]^{-1}\right. \\
& \left.+128 \varepsilon^{2}\left(x^{2}+r^{2}+C\right)^{-2}\left(x^{2}+r^{2}\right)\left(3 x^{2}+3 r^{2}+C\right)\left[\sin (A t+B)+\frac{3}{2}\right]^{2}\right\}, \\
f_{2}(x, r, t, \varepsilon)= & \frac{1}{2} \varepsilon^{2} A\left(x^{2}+r^{2}+C\right)^{\frac{1}{2}}\left[\sin (A t+B)+\frac{3}{2}\right]^{-\frac{1}{2}} \cos (A t+B) .
\end{aligned}
$$

Assume homogeneous Neumann condition on the lower boundary, and homogeneous Dirichlet condition on the other boundaries. The exact solution is $T=\left(x^{2}+r^{2}+\right.$ $C)^{\frac{1}{2}}\left[\sin (A t+B)+\frac{3}{2}\right]^{\frac{1}{2}}, E=\left(x^{2}+r^{2}+C\right)^{2}\left[\sin (A t+B)+\frac{3}{2}\right]^{2}$.

Table 2 illustrates the numerical accuracy of energy and temperature at $t=0.1$ with $\lambda=0.8$. Herein we take $A=\frac{1}{2}+\frac{1}{2} \varepsilon, B=\frac{1}{4}+\frac{1}{4} \varepsilon$ and $C=\frac{1}{4}+\frac{1}{4} \varepsilon$ with $\varepsilon=1.0$. It confirms our 
Table 2: Numerical accuracy of energy and temperature for Example 5.4.

\begin{tabular}{||ccccccc||}
\hline scheme & mesh & $8 \times 8$ & $16 \times 16$ & $32 \times 32$ & $64 \times 64$ & order \\
\hline a-Bnd1 & error-E & $3.16140 \mathrm{e}-1$ & $1.43906 \mathrm{e}-1$ & $6.33243 \mathrm{e}-2$ & $2.58477 \mathrm{e}-2$ & 1.20415 \\
& error-T & $6.26703 \mathrm{e}-3$ & $2.80127 \mathrm{e}-3$ & $1.25224 \mathrm{e}-3$ & $5.21854 \mathrm{e}-4$ & 1.19535 \\
\hline b-Bnd1 & error-E & $3.07663 \mathrm{e}-1$ & $1.40196 \mathrm{e}-1$ & $6.23468 \mathrm{e}-2$ & $2.55735 \mathrm{e}-2$ & 1.19621 \\
& error-T & $6.01473 \mathrm{e}-3$ & $2.72490 \mathrm{e}-3$ & $1.23241 \mathrm{e}-3$ & $5.16121 \mathrm{e}-4$ & 1.18091 \\
\hline \multirow{2}{*}{ c-Bnd1 } & error-E & $2.90300 \mathrm{e}-1$ & $1.42022 \mathrm{e}-1$ & $6.50832 \mathrm{e}-2$ & $2.65307 \mathrm{e}-2$ & 1.15060 \\
& error-T & $5.79617 \mathrm{e}-3$ & $2.80578 \mathrm{e}-3$ & $1.30262 \mathrm{e}-3$ & $5.39738 \mathrm{e}-4$ & 1.14159 \\
\hline a-Bnd2 & error-E & $1.33787 \mathrm{e}-1$ & $3.29249 \mathrm{e}-2$ & $8.06861 \mathrm{e}-3$ & $1.96754 \mathrm{e}-3$ & 2.02913 \\
& error-T & $4.73797 \mathrm{e}-3$ & $1.13443 \mathrm{e}-3$ & $2.84326 \mathrm{e}-4$ & $7.09406 \mathrm{e}-5$ & 2.02051 \\
\hline b-Bnd2 & error-E & $1.25742 \mathrm{e}-1$ & $3.10775 \mathrm{e}-2$ & $7.58797 \mathrm{e}-3$ & $1.84673 \mathrm{e}-3$ & 2.02978 \\
& error-T & $4.14687 \mathrm{e}-3$ & $9.83102 \mathrm{e}-4$ & $2.44145 \mathrm{e}-4$ & $6.08721 \mathrm{e}-5$ & 2.03003 \\
\hline c-Bnd2 & error-E & $5.40569 \mathrm{e}-2$ & $1.75176 \mathrm{e}-2$ & $5.02465 \mathrm{e}-3$ & $1.18245 \mathrm{e}-3$ & 1.83821 \\
& error-T & $1.42962 \mathrm{e}-3$ & $3.33560 \mathrm{e}-4$ & $7.91853 \mathrm{e}-5$ & $1.94559 \mathrm{e}-5$ & 2.06643 \\
\hline ap-Bnd1 & error-E & $4.12699 \mathrm{e}-1$ & $1.86933 \mathrm{e}-1$ & $7.70108 \mathrm{e}-2$ & $3.00573 \mathrm{e}-2$ & 1.25977 \\
& error-T & $1.01553 \mathrm{e}-2$ & $3.89534 \mathrm{e}-3$ & $1.56813 \mathrm{e}-3$ & $6.25086 \mathrm{e}-4$ & 1.21801 \\
\hline bp-Bnd1 & error-E & $4.03268 \mathrm{e}-1$ & $1.83133 \mathrm{e}-1$ & $7.58687 \mathrm{e}-2$ & $2.97805 \mathrm{e}-2$ & 1.25310 \\
& error-T & $9.59699 \mathrm{e}-3$ & $3.81629 \mathrm{e}-3$ & $1.54736 \mathrm{e}-3$ & $6.19279 \mathrm{e}-4$ & 1.31797 \\
\hline cp-Bnd1 & error-E & $3.86986 \mathrm{e}-1$ & $1.85064 \mathrm{e}-1$ & $7.79779 \mathrm{e}-2$ & $3.07415 \mathrm{e}-2$ & 1.21801 \\
& error-T & $8.07454 \mathrm{e}-3$ & $3.90334 \mathrm{e}-3$ & $1.61808 \mathrm{e}-3$ & $6.43053 \mathrm{e}-4$ & 1.21679 \\
\hline ap-Bnd2 & error-E & $2.55663 \mathrm{e}-1$ & $7.97832 \mathrm{e}-2$ & $2.37343 \mathrm{e}-2$ & $6.82070 \mathrm{e}-3$ & 1.74273 \\
& error-T & $9.79132 \mathrm{e}-3$ & $3.05514 \mathrm{e}-3$ & $9.33932 \mathrm{e}-4$ & $2.76544 \mathrm{e}-4$ & 1.71531 \\
\hline bp-Bnd2 & error-E & $2.48043 \mathrm{e}-1$ & $7.79046 \mathrm{e}-2$ & $2.32527 \mathrm{e}-2$ & $6.70361 \mathrm{e}-3$ & 1.73650 \\
& error-T & $9.14776 \mathrm{e}-3$ & $2.87347 \mathrm{e}-3$ & $8.87094 \mathrm{e}-4$ & $2.64533 \mathrm{e}-4$ & 1.70397 \\
\hline cp-Bnd2 & error-E & $1.57483 \mathrm{e}-1$ & $6.17811 \mathrm{e}-2$ & $1.96298 \mathrm{e}-2$ & $5.71421 \mathrm{e}-3$ & 1.59483 \\
& error-T & $3.79126 \mathrm{e}-3$ & $1.69783 \mathrm{e}-3$ & $6.07963 \mathrm{e}-4$ & $1.96851 \mathrm{e}-4$ & 1.42250 \\
\hline
\end{tabular}

schemes with Choice 1 and Bnd2 have second order accuracy in the mass, which perform much better than schemes with Bnd1 with obvious decrease of the error data. In this test, they look even better than those with Bnd2 and familiar physical harmonic average Choice 2. It may be reasonable considering the global treatment of diffusion coefficient of Choice 1 since we measure the maximum errors on the whole domain instead of only near the polar axis. Also their convergence performance may be influenced by the parameters of the concrete coefficient and source terms and the spatial and temporal step lengths. The later seems to converge with an order around 1.5-1.7 instead of 2. It is not strange anyway, since $\frac{\partial E}{\partial r} \neq 0$ on the upper boundary, we know from Appendix B the error of the IB schemes with Choice 2 is $\mathcal{O}\left(\varepsilon^{2} h+h^{2}+\tau\right)$ at the points adjacent to this boundary, which may affect the global accuracy accordingly. There are other tests show they have comparable results. 
Example 5.5. Consider (5.3) with homogeneous Dirichlet condition on the left, right and upper boundaries, and homogeneous Neumann condition on the lower boundary. Its exact solution is $T=\sin \theta, E=\sin ^{4} \theta$. The additional terms can be written accordingly, i.e.,

$$
\begin{aligned}
f_{1}(x, r, t, \varepsilon)= & 4 \varepsilon^{2} A \sin ^{3} \theta \cos \theta \\
& -8 \varepsilon^{2}\left[9 \sin ^{-6} \theta+64 \varepsilon^{2}\left(x^{2}+r^{2}\right) \cot ^{2} \theta\right]^{-\frac{3}{2}} \\
& {\left[108\left(x^{2}+r^{2}\right) \sin ^{-4} \theta \cos ^{2} \theta+128 \varepsilon^{2}\left(x^{2}+r^{2}\right) \sin \theta \cos ^{3} \theta\right.} \\
& \left.+512 \varepsilon^{2}\left(x^{2}+r^{2}\right)^{2} \cos ^{4} \theta+27 \sin ^{-3} \theta \cos \theta-18\left(x^{2}+r^{2}\right) \sin ^{-2} \theta\right], \\
f_{2}(x, r, t, \varepsilon)= & \varepsilon^{2} A \cos \theta .
\end{aligned}
$$

We take $\theta=x^{2}+r^{2}+A t+B, A=2+\varepsilon, B=\frac{1}{3}+\frac{1}{3} \varepsilon$, and measure the numerical errors at $t=0.1$ with $\lambda=0.64$ and various $\varepsilon$. They all converge at about order 2 . As examples, Fig. 4 depicts the results with $\varepsilon=1$ and $10^{-12}$.
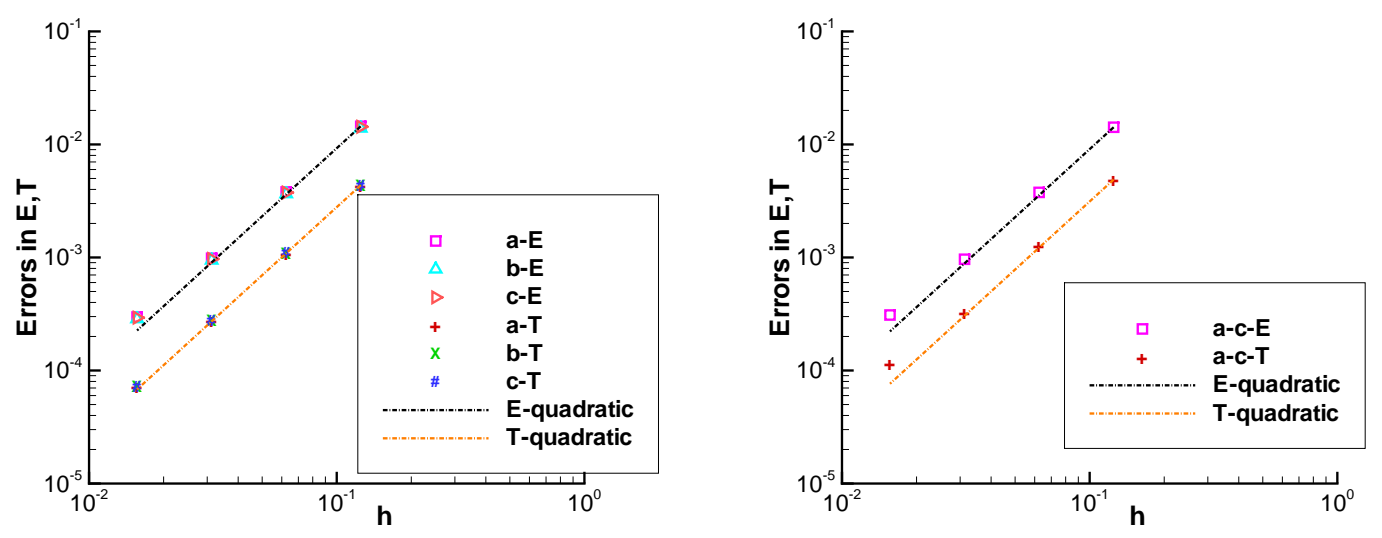

Figure 4: Numerical accuracy of energy and temperature with $\varepsilon=1$ (left) and $10^{-12}$ (right) for Example 5.5.

Finally, we examine the asymptotic-preserving property of the schemes by using reference solutions. We briefly review some conceptions here to ease the description later [16]. Let $T, E, T^{(0)}, E^{(0)}$ and $T^{(1)}, E^{(1)}$ respectively stand for the solutions of the discrete scaled equation and its zero-th and first order discrete asymptotic equations. And define $T-T^{(0)}, E-E^{(0)}$ and $T-\left[T^{(0)}+\varepsilon T^{(1)}\right], E-\left[E^{(0)}+\varepsilon E^{(1)}\right]$ as the zero-th and first order discrete asymptotic errors correspondingly. When $\varepsilon$ tends to 0 , if the zero-th and first order discrete asymptotic errors converge as $\mathcal{O}(\varepsilon)$ and $\mathcal{O}\left(\varepsilon^{2}\right)$ respectively, then the scheme is first order asymptotic-preserving.

Example 5.6. Consider the scaled spherical non-equilibrium radiation diffusion problem (2.2) in Example 5.3.

We take $\varepsilon$ as $1 / 100,1 / 200,1 / 400$ and $1 / 800$, and still use the numerical solutions with $h=1 / 3200$ as reference solutions. Figs. 5 and 6 respectively display the maximum norm 

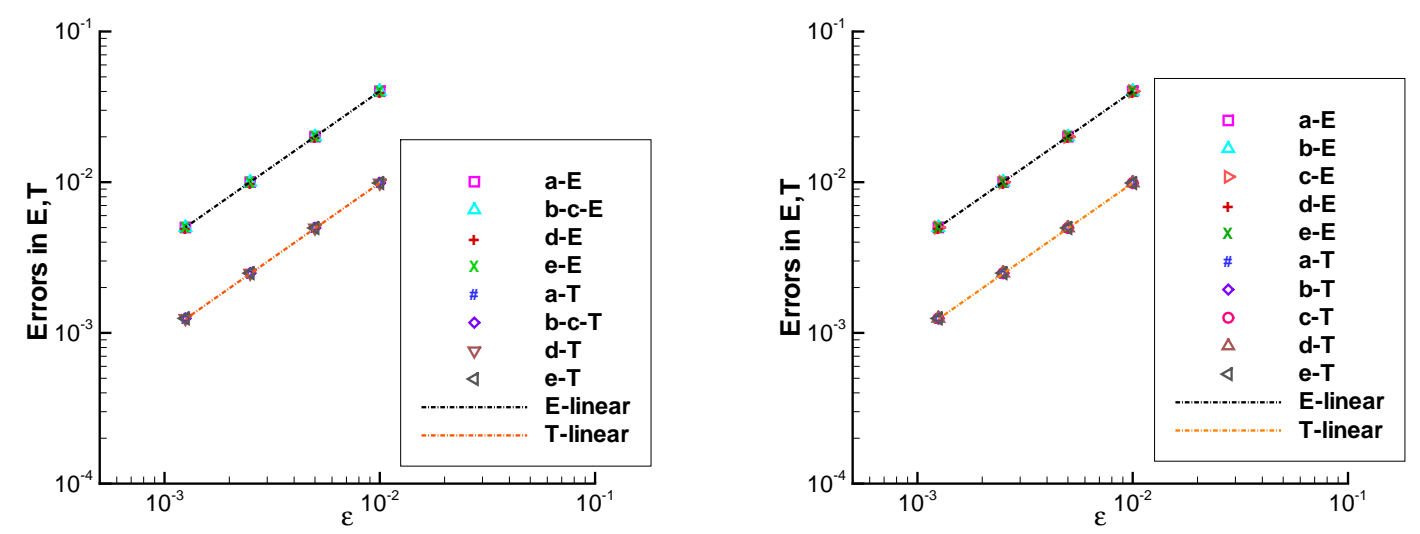

Figure 5: The zero-th order discrete asymptotic errors with $h=1 / 50$ (left) and $h=1 / 3200$ (right) for Example 5.6.
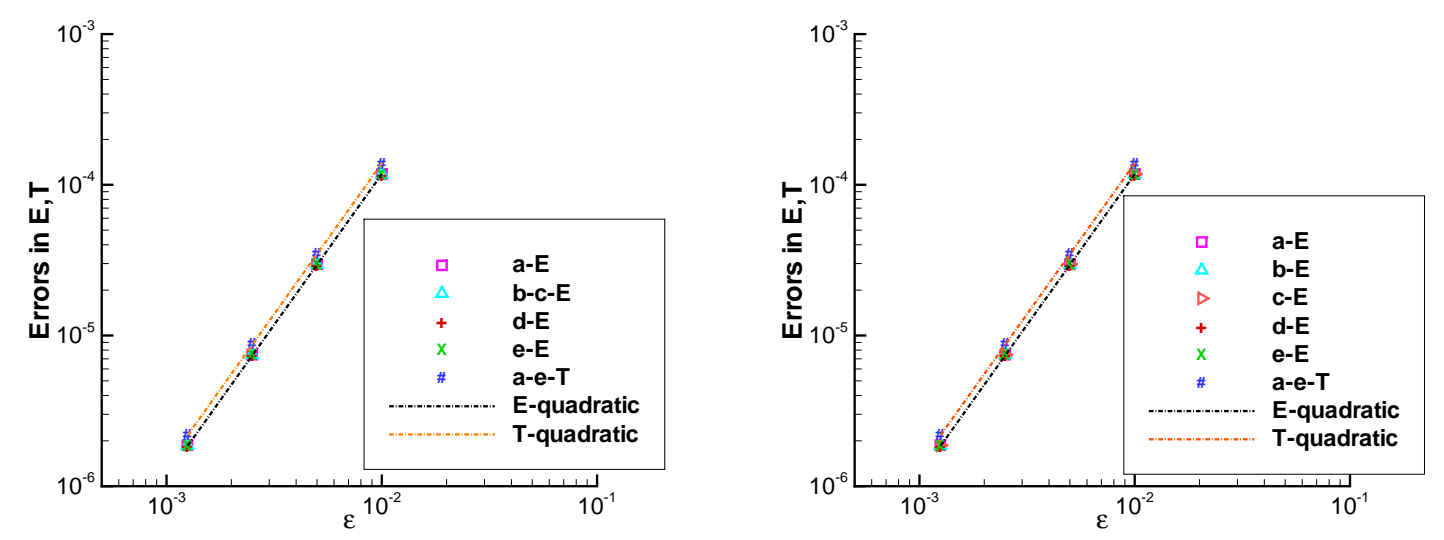

Figure 6: The first order discrete asymptotic errors with $h=1 / 50$ (left) and $h=1 / 3200$ (right) for Example 5.6.

zero-th and first order discrete asymptotic errors for energy and temperature at $t=0.1$ with various spatial steps (e.g., $h=1 / 50$ and 1/3200). Evidently they converge at order 1 and 2 respectively. Moreover, the convergence behavior is independent of the time and spatial steps as the scaling parameter tends to zero. Hence the schemes are first order asymptotic-preserving as theoretical prediction, and adaptive to equilibrium as well as non-equilibrium radiation diffusion problems.

Example 5.7. We use reference solutions to test the asymptotic property of the cylindrical schemes. Consider the scaled non-equilibrium radiation diffusion problem (4.1)-(4.2). A group of boundary and initial conditions of its zero-th order asymptotic equation (4.3) 
read

$$
\begin{aligned}
& E^{(0)}(0, r, t)=\frac{1}{6}\left(\frac{r}{3} e^{-r t}-\frac{r}{3}+1\right), \quad E^{(0)}(1, r, t)=\left(e^{-1}+\frac{1}{6}\right)\left(\frac{r}{3} e^{-r t}-\frac{r}{3}+1\right) ; \\
& \frac{\partial E^{(0)}}{\partial r}(x, 0, t)=0, \quad E^{(0)}(x, 1, t)=\left(\frac{1}{3} e^{-t}+\frac{2}{3}\right)\left(x e^{-x}+\frac{1}{6}\right) \\
& T^{(0)}(x, r, 0)=\left(x e^{-x}+\frac{1}{6}\right)^{\frac{1}{4}}, \quad E^{(0)}(x, r, 0)=x e^{-x}+\frac{1}{6} .
\end{aligned}
$$

Those of its first order asymptotic equation (4.4) are

$$
\begin{aligned}
& E^{(1)}(0, r, t)=\left(\frac{1}{3}\right)^{\frac{1}{2}}\left(2 r e^{-r t}-2 r+\frac{20}{3}\right), \quad E^{(1)}(1, r, t)=\left(e^{-1}+\frac{1}{3}\right)^{\frac{1}{2}}\left(2 r e^{-r t}-2 r+\frac{20}{3}\right) \\
& \frac{\partial E^{(1)}}{\partial r}(x, 0, t)=0, \quad E^{(1)}(x, 1, t)=\left(2 e^{-t}+\frac{14}{3}\right)\left(x e^{-x}+\frac{1}{3}\right)^{\frac{1}{2}} \\
& T^{(1)}(x, r, 0)=\frac{5}{3}\left(x e^{-x}+\frac{1}{3}\right)^{\frac{1}{2}}\left(x e^{-x}+\frac{1}{6}\right)^{-\frac{3}{4}}, \quad E^{(1)}(x, r, 0)=\frac{20}{3}\left(x e^{-x}+\frac{1}{3}\right)^{\frac{1}{2}}
\end{aligned}
$$

Correspondingly, we take $T^{(0)}+\varepsilon T^{(1)}$ and $E^{(0)}+\varepsilon E^{(1)}$ as initial and boundary conditions of (4.1).

We take the numerical solutions obtained on a $128 \times 128$ spatial mesh as reference solutions. This is not an ideal choice since the mesh is still rough anyway. But with its help we can show the errors are decreasing as the mesh becomes finer, which is a hint of convergence. A favorable fact is that for asymptotic test with discrete asymptotic errors, the reference solutions are not necessary. Fig. 7 exhibits the maximum zero-th and first order discrete asymptotic errors of energy and temperature on a $32 \times 32$ mesh. Evidently they are first and second order convergent respectively. There are similar results with other spatial and temporal steps. Hence we argue the schemes are first order asymptoticpreserving.

\section{Conclusion and perspectives}

We study the fully discrete AP schemes for non-equilibrium radiation diffusion problems in one-dimensional spherical symmetric geometry, and extend the results to problems in two-dimensional cylindrical symmetric geometry. In the scheme design, we apply finite volume method to handle the singularity at the origin and the polar axis and to guarantee the local conservative property, in which asymmetric second order accurate spatial approach is used for the boundary flux-limiters in the diffusion operators, and discrete schemes with higher order global accuracy are attained. By formal analysis, we prove these schemes are first order asymptotic-preserving. By contriving artificial 

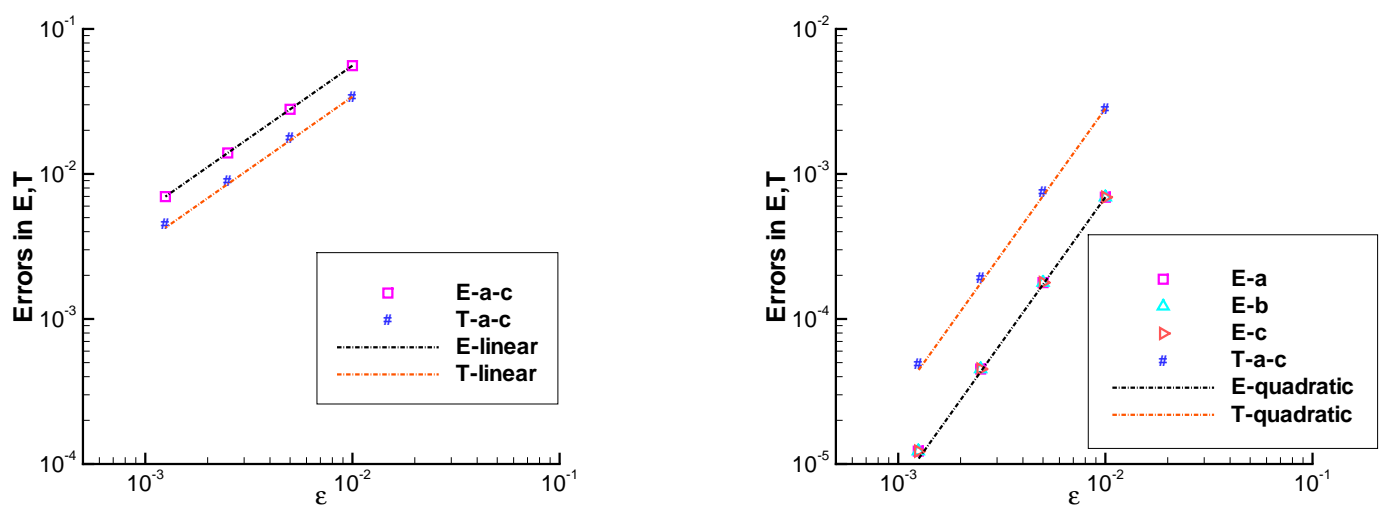

Figure 7: The zero-th (left) and first (right) order discrete asymptotic errors for Example 5.7.

solutions, we quantitatively validate the accuracy and asymptotic-preserving property of the schemes with numerical tests, and confirm their applicability for both equilibrium and non-equilibrium radiation diffusion problems.

In Appendix A, the second order accuracy of the harmonic average in the spherical coordinate system is demonstrated. Containing the geometry feature, the discrete diffusive coefficient differs slightly from the familiar average obtained from pure physics flux continuity, but it gives similar and better numerical results in numerical tests. However, it is frustrating that we can only show the first order instead of the second order accuracy of the harmonic average near the polar axis in the corresponding cylindrical case. Maybe there are some delicate details or other tools to weed through the confusion we are not aware of. Or the schemes are not strictly second order in theory. Nevertheless this will not influence the AP property of the cylindrical schemes even with only first order spatial accuracy theoretically. As a supplement, we also present the truncation error of the discrete diffusion operators in Appendix B.

The study can be extended from first order temporal accurate schemes (i.e., schemes with $\mathcal{O}(\tau)$ temporal accuracy as $\tau \rightarrow 0)$ to second order $\left(\mathcal{O}\left(\tau^{2}\right)\right)$ temporal accurate implicit schemes. For multi-dimensional (cylindrical) problems, a natural way to accelerate their solutions is to use splitting algorithms such as operator-splitting discretizations or fractional-step time evolutions [29]. In this case, it is delicate to design the splitting strategy and boundary treatment for each splitting step to acquire perfect global consistent error to construct AP schemes. Also the ideas contribute to studying AP schemes on skewed and unstructured meshes.

\section{Acknowledgments}

The authors are very grateful to the editors and the anonymous referees for helpful suggestions to enhance the paper. This work is supported by the National Natural Science 
Foundation of China (11271054, 11471048, 11571048, U1630249), the Science Foundation of CAEP (2014A0202010), the Science Challenge Project (No. JCKY2016212A502) and the Foundation of LCP.

\section{Appendix A}

This appendix is devoted to demonstrate the second order accuracy for harmonic average approach in spherical geometry.

Let $u$ be a continuous function with enough smoothness. In the following, denote

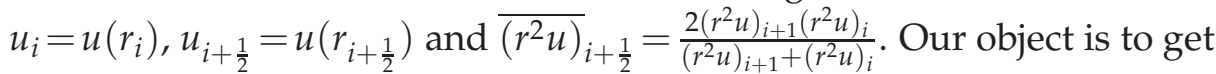

$$
{\overline{\left(r^{2} u\right)_{i+\frac{1}{2}}}}=\left(r^{2} u\right)_{i+\frac{1}{2}}+\mathcal{O}\left(h^{2}\right) .
$$

We start from Taylor's expansion,

$$
\begin{aligned}
& \left(r^{2} u\right)_{i+1}=\left(r^{2} u\right)_{i+\frac{1}{2}}+\frac{h}{2}\left(r^{2} u\right)_{r i+\frac{1}{2}}^{\prime}+\frac{h^{2}}{8}\left(r^{2} u\right)_{r r i+\frac{1}{2}}^{\prime \prime}+\frac{h^{3}}{48}\left(r^{2} u\right)_{r r r i+\frac{1}{2}}^{\prime \prime \prime}+\mathcal{O}\left(h^{4}\right), \\
& \left(r^{2} u\right)_{i}=\left(r^{2} u\right)_{i+\frac{1}{2}}-\frac{h}{2}\left(r^{2} u\right)_{r i+\frac{1}{2}}^{\prime}+\frac{h^{2}}{8}\left(r^{2} u\right)_{r r i+\frac{1}{2}}^{\prime \prime}-\frac{h^{3}}{48}\left(r^{2} u\right)_{r r r+\frac{1}{2}}^{\prime \prime \prime}+\mathcal{O}\left(h^{4}\right),
\end{aligned}
$$

therefore

$$
\begin{aligned}
\left(r^{2} u\right)_{i+1}\left(r^{2} u\right)_{i}= & {\left[\left(r^{2} u\right)_{i+\frac{1}{2}}+\frac{h^{2}}{8}\left(r^{2} u\right)_{r r i+\frac{1}{2}}^{\prime \prime}\right]^{2}-\left[\frac{h}{2}\left(r^{2} u\right)_{r i+\frac{1}{2}}^{\prime}+\frac{h^{3}}{48}\left(r^{2} u\right)_{r r r i+\frac{1}{2}}^{\prime \prime \prime}\right]^{2} } \\
& +\mathcal{O}\left(h^{4}\right)\left[\left(r^{2} u\right)_{i+\frac{1}{2}}+\frac{h}{2}\left(r^{2} u\right)_{r i+\frac{1}{2}}^{\prime}+\mathcal{O}\left(h^{2}\right)\right] \\
\left(r^{2} u\right)_{i+1}+\left(r^{2} u\right)_{i}= & 2\left(r^{2} u\right)_{i+\frac{1}{2}}+\frac{h^{2}}{4}\left(r^{2} u\right)_{r r i+\frac{1}{2}}^{\prime \prime}+\mathcal{O}\left(h^{4}\right) .
\end{aligned}
$$

Noticing that

$$
\begin{aligned}
& \left(r^{2} u\right)_{r}^{\prime}=2 r u+r^{2} u_{r}^{\prime},\left(r^{2} u\right)_{r r}^{\prime \prime}=2 u+4 r u_{r}^{\prime}+r^{2} u_{r r}^{\prime \prime}, \\
& \left(r^{2} u\right)_{r r r}^{\prime \prime \prime}=6 u_{r}^{\prime}+6 r u_{r r}^{\prime \prime}+r^{2} u_{r r}^{\prime \prime \prime},
\end{aligned}
$$

we arrive at

$$
{\overline{\left(r^{2} u\right)_{i+\frac{1}{2}}}}=\left(r^{2} u\right)_{i+\frac{1}{2}} \frac{I_{1}}{I_{2}}
$$

where

$$
\begin{aligned}
& I_{1}=\left[1+\frac{h^{2}}{8} \frac{\left(r^{2} u\right)_{r r i+\frac{1}{2}}^{\prime \prime}}{\left(r^{2} u\right)_{i+\frac{1}{2}}}\right]^{2}-\left[\frac{h}{2} \frac{\left(r^{2} u\right)_{r i+\frac{1}{2}}^{\prime}}{\left(r^{2} u\right)_{i+\frac{1}{2}}}+\frac{h^{3}}{48} \frac{\left(r^{2} u\right)_{r r r i+\frac{1}{2}}^{\prime \prime \prime}}{\left(r^{2} u\right)_{i+\frac{1}{2}}}\right]^{2}+\mathcal{O}\left(h^{2}\right), \\
& I_{2}=1+\frac{h^{2}}{8} \frac{\left(r^{2} u\right)_{r r i+\frac{1}{2}}^{\prime \prime}}{\left(r^{2} u\right)_{i+\frac{1}{2}}}+\mathcal{O}\left(h^{2}\right) .
\end{aligned}
$$


When $r$ lies far from the origin, i.e., $r \gg h$, it is obvious that

$$
I_{1}=1+\mathcal{O}\left(h^{2}\right), \quad I_{2}=1+\mathcal{O}\left(h^{2}\right),
$$

hence (A.1) holds.

When $r$ lies near the origin, $r=\mathcal{O}(h)$, there stands

$$
\begin{aligned}
\frac{1}{I_{2}} & =\left[1+\frac{h^{2}}{8} \frac{\left(r^{2} u\right)_{r r i+\frac{1}{2}}^{\prime \prime}}{\left(r^{2} u\right)_{i+\frac{1}{2}}}\right]^{-1}\left\{1-\left[1+\frac{h^{2}}{8} \frac{\left(r^{2} u\right)_{r r i+\frac{1}{2}}^{\prime \prime}}{\left(r^{2} u\right)_{i+\frac{1}{2}}}\right]^{-1} \mathcal{O}\left(h^{2}\right)+\mathcal{O}\left(h^{4}\right)\right\} \\
& =\left[1+\frac{h^{2}}{8} \frac{\left(r^{2} u\right)_{r r i+\frac{1}{2}}^{\prime \prime}}{\left(r^{2} u\right)_{i+\frac{1}{2}}}\right]^{-1}\left[1+\mathcal{O}\left(h^{2}\right)\right] .
\end{aligned}
$$

We have

$$
\begin{aligned}
& \left(r^{2} u\right)\left[\frac{h}{2} \frac{\left(r^{2} u\right)_{r}^{\prime}}{\left(r^{2} u\right)}+\frac{h^{3}}{48} \frac{\left(r^{2} u\right)_{r r r}^{\prime \prime \prime}}{\left(r^{2} u\right)}\right]^{2} \\
= & \frac{1}{u}\left[h u+\frac{h r}{2} u_{r}^{\prime}+\frac{h^{3}}{8 r} u_{r}^{\prime}+\frac{h^{3}}{8} u_{r r}^{\prime \prime}+\frac{h^{3} r}{48} u_{r r r}^{\prime \prime \prime}\right]^{2}=\mathcal{O}\left(h^{2}\right) .
\end{aligned}
$$

Combining (A.2), (A.3) and (A.4), we get

$$
\begin{aligned}
{\overline{\left(r^{2} u\right)_{i+\frac{1}{2}}}} & \left(r^{2} u\right)_{i+\frac{1}{2}}+\frac{h^{2}}{8}\left(r^{2} u\right)_{r r i+\frac{1}{2}}^{\prime \prime}-\mathcal{O}\left(h^{2}\right)\left[1+\mathcal{O}\left(h^{2}\right)\right]+\mathcal{O}\left(h^{2}\right) \\
& =\left(r^{2} u\right)_{i+\frac{1}{2}}+\mathcal{O}\left(h^{2}\right)
\end{aligned}
$$

Hence the conclusion is true.

A similar deduction shows the first order accuracy for harmonic average approach in cylindrical geometry near the polar axis.

\section{Appendix B}

In this appendix we demonstrate the truncation error of the discrete diffusion operators.

By a lengthy manipulation, we derive the truncation error as follows

$$
\begin{aligned}
G_{i} & =\frac{1}{h}\left(\frac{2 \hat{d}_{i+1} \hat{d}_{i}}{\hat{d}_{i+1}+\hat{d}_{i}} \frac{E_{i+1}-E_{i}}{h}-\frac{2 \hat{d}_{i} \hat{d}_{i-1}}{\hat{d}_{i}+\hat{d}_{i-1}} \frac{E_{i}-E_{i-1}}{h}\right)-\left(d E^{\prime}\right)_{i}^{\prime} \\
& =Q_{i}+\mathcal{O}\left(h^{2}\right)+\mathcal{O}\left(\varepsilon^{2} h^{2}\right), \quad i=2,3, \cdots, I-3, I-2,
\end{aligned}
$$




$$
\begin{aligned}
& G_{1}=\frac{1}{h}\left(\frac{2 \hat{d}_{i+1} \hat{d}_{i}}{\hat{d}_{i+1}+\hat{d}_{i}} \frac{E_{i+1}-E_{i}}{h}-\frac{2 \hat{d}_{i} \tilde{d}_{i-1}}{\hat{d}_{i}+\tilde{d}_{i-1}} \frac{E_{i}-E_{i-1}}{h}\right)-\left(d E^{\prime}\right)_{i}^{\prime} \\
& =Q_{1}+\frac{1}{h} \frac{2 \hat{d}_{i}^{2}\left(\hat{d}_{i-1}-\tilde{d}_{i-1}\right)}{\left(\hat{d}_{i}+\hat{d}_{i-1}\right)\left(\hat{d}_{i}+\tilde{d}_{i-1}\right)} E_{\frac{1}{2}}^{\prime}+\mathcal{O}\left(h^{2}\right)+\mathcal{O}\left(\varepsilon^{2} h^{2}\right) \\
& = \begin{cases}Q_{1}+\mathcal{O}\left(\varepsilon^{2}\right) E_{\frac{1}{2}}^{\prime}+\mathcal{O}\left(h^{2}\right)+\mathcal{O}\left(\varepsilon^{2} h^{2}\right), & \text { with Bnd1; } \\
Q_{1}+\mathcal{O}\left(\varepsilon^{2} h\right) E_{\frac{1}{2}}^{\prime}+\mathcal{O}\left(h^{2}\right)+\mathcal{O}\left(\varepsilon^{2} h^{2}\right), & \text { with Bnd2, }\end{cases} \\
& G_{I-1}=\frac{1}{h}\left(\frac{2 \tilde{d}_{i+1} \hat{d}_{i}}{\tilde{d}_{i+1}+\hat{d}_{i}} \frac{E_{i+1}-E_{i}}{h}-\frac{2 \hat{d}_{i} \hat{d}_{i-1}}{\hat{d}_{i}+\hat{d}_{i-1}} \frac{E_{i}-E_{i-1}}{h}\right)-\left(d E^{\prime}\right)_{i}^{\prime} \\
& =Q_{I-1}-\frac{1}{h} \frac{2 \hat{d}_{i}^{2}\left(\hat{d}_{i+1}-\tilde{d}_{i+1}\right)}{\left(\hat{d}_{i+1}+\hat{d}_{i}\right)\left(\tilde{d}_{i+1}+\hat{d}_{i}\right)} E_{I-\frac{1}{2}}^{\prime}+\mathcal{O}\left(h^{2}\right)+\mathcal{O}\left(\varepsilon^{2} h^{2}\right) \\
& = \begin{cases}Q_{I-1}+\mathcal{O}\left(\varepsilon^{2}\right) E_{I-\frac{1}{2}}^{\prime}+\mathcal{O}\left(h^{2}\right)+\mathcal{O}\left(\varepsilon^{2} h^{2}\right), & \text { with Bnd1; } \\
Q_{I-1}+\mathcal{O}\left(\varepsilon^{2} h\right) E_{I-\frac{1}{2}}^{\prime}+\mathcal{O}\left(h^{2}\right)+\mathcal{O}\left(\varepsilon^{2} h^{2}\right), & \text { with Bnd2, }\end{cases}
\end{aligned}
$$

where

$$
\begin{aligned}
Q_{i}= & \left\{\frac{h^{2}}{4}\left[\left(d^{\prime}\right)^{3} E^{\prime}\right]_{i}-\frac{h^{2}}{4}\left[\left(d^{\prime}\right)^{2} E^{\prime}\right]_{i}^{\prime} d_{i}+\frac{3 h^{4}}{48}\left[\left(d^{\prime}\right)^{2} d^{\prime \prime \prime} E^{\prime}\right]_{i}+\frac{h^{4}}{32}\left[\left(d^{\prime}\right)^{2} E^{\prime}\right]_{i}^{\prime \prime} d_{i}^{\prime}\right. \\
& \left.-\frac{h^{4}}{16}\left[\left(d^{\prime}\right)^{2} E^{\prime}\right]_{i}^{\prime} d_{i}^{\prime \prime}-\frac{h^{4}}{48}\left(d^{\prime} d^{\prime \prime \prime} E^{\prime}\right)_{i}^{\prime} d_{i}-\frac{h^{4}}{96}\left[\left(d^{\prime}\right)^{2} E^{\prime}\right]_{i}^{\prime \prime \prime} d_{i}+\mathcal{O}\left(h^{6}\right)\right\} \\
& \left\{d_{i}^{2}+h^{2}\left[\frac{1}{2} d_{i} d_{i}^{\prime \prime}-\frac{1}{4}\left(d_{i}^{\prime}\right)^{2}\right]+\mathcal{O}\left(h^{4}\right)\left(d_{i}+d_{i}^{\prime} d_{i}^{\prime \prime \prime}\right)+\mathcal{O}\left(h^{4}\right)+\mathcal{O}\left(h^{6}\right)\right\}^{-1},
\end{aligned}
$$

and $\hat{d}_{i}=D_{R i}$, or $r D_{R i}$ or $r^{2} D_{R i}$ is defined as in Sections 2 and 4 . For $i=1$ and $I-1, \tilde{d}_{i}=\widetilde{D}_{R i}$, or $\widetilde{r D}_{R i}$ or $\widetilde{r}^{2} D_{R i}$, where $\widetilde{r D}_{R i}=\frac{r_{i}}{\sqrt{(3 \sigma)_{i}^{2}+\varepsilon^{2} \tilde{\chi}_{i}}}$, etc.

We denote $Q_{i}$ as $Q_{s i}$ or $Q_{c i}$ in the case of spherical or cylindric symmetric geometry. Near the origin or symmetric axis, generally, $Q_{s i}=\mathcal{O}\left(h+\varepsilon^{2} h^{2}\right), Q_{c i}=\mathcal{O}\left(1+\varepsilon^{2} h\right)$; elsewhere $Q_{i}=\mathcal{O}\left(h^{2}+\varepsilon^{2} h^{2}\right)$. When $E_{0}^{\prime}=0$, we have $Q_{s i}=\mathcal{O}\left(h^{2}+\varepsilon^{2} h^{3}\right)$ or $Q_{c i}=\mathcal{O}\left(h+\varepsilon^{2} h^{2}\right)$ near the origin or symmetric axis, and $E_{\frac{1}{2}}^{\prime}=\mathcal{O}(h)$. When $E_{I}^{\prime}=0$, we have $E_{I-\frac{1}{2}}^{\prime}=\mathcal{O}(h)$.

Combining these acquaintances, we present the truncation error of the discrete diffusion operator (corresponding to Choice 1 in the sphere and cylinder cases) in Table 3.

In the case of Choice 2, e.g., the familiar physical harmonic average as (2.15) and (2.16) in the sphere case and as described in Remark 4.2 in the cylinder case, the truncation error order for discrete diffusion operator is analogous to that in planar geometry. Hence theoretically, with Bnd2 and homogeneous Neumann boundary condition (2.4), the two ways of harmonic average deliver comparable accuracy in the sphere case, and the physical harmonic average (Choice 2) seems more precise than cylindrical harmonic average (Choice 1) in the cylinder case near the polar axis. Our numerical tests coincide well with 
Table 3: Truncation error for discrete diffusion operator.

\begin{tabular}{|c|c|c|c|c|c|c|}
\hline$G_{i}$ & Plane-Bnd1 & Plane-Bnd2 & Sphere-Bnd1 & Sphere-Bnd2 & Cylinder-Bnd1 & Cylinder-Bnd2 \\
\hline $\begin{array}{l}i \neq 1, I-1 \\
\text { 1) } i \gg 1 \\
\text { 2) otherwise } \\
\text { 2.1) } E_{0}^{\prime}=0 \\
\text { 2.2) } E_{0}^{\prime} \neq 0 \\
\end{array}$ & \multicolumn{2}{|c|}{$\mathcal{O}\left(h^{2}+\varepsilon^{2} h^{2}\right)$} & \multicolumn{2}{|c|}{$\begin{array}{c}\mathcal{O}\left(h^{2}+\varepsilon^{2} h^{2}\right) \\
\mathcal{O}\left(h^{2}+\varepsilon^{2} h^{2}\right) \\
\mathcal{O}\left(h+\varepsilon^{2} h^{2}\right)\end{array}$} & \multicolumn{2}{|c|}{$\begin{array}{c}\mathcal{O}\left(h^{2}+\varepsilon^{2} h^{2}\right) \\
\mathcal{O}\left(h+\varepsilon^{2} h^{2}\right) \\
\mathcal{O}(1)\end{array}$} \\
\hline $\begin{array}{l}i=1 \\
\text { 1) } E_{0}^{\prime}=0 \\
\text { 2) } E_{0}^{\prime} \neq 0\end{array}$ & $\begin{array}{c}\mathcal{O}\left(\varepsilon^{2} h+h^{2}\right) \\
\mathcal{O}\left(\varepsilon^{2}+h^{2}\right)\end{array}$ & $\begin{array}{c}\mathcal{O}\left(h^{2}+\varepsilon^{2} h^{2}\right) \\
\mathcal{O}\left(\varepsilon^{2} h+h^{2}\right)\end{array}$ & $\begin{array}{c}\mathcal{O}\left(\varepsilon^{2} h+h^{2}\right) \\
\mathcal{O}\left(\varepsilon^{2}+h\right)\end{array}$ & $\begin{array}{c}\mathcal{O}\left(h^{2}+\varepsilon^{2} h^{2}\right) \\
\mathcal{O}\left(h+\varepsilon^{2} h\right)\end{array}$ & $\begin{array}{c}\mathcal{O}\left(h+\varepsilon^{2} h\right) \\
\mathcal{O}(1)\end{array}$ & $\begin{array}{c}\mathcal{O}\left(h+\varepsilon^{2} h^{2}\right) \\
\mathcal{O}(1)\end{array}$ \\
\hline $\begin{array}{l}i=I-1 \\
\text { 1) } E_{I}^{\prime}=0 \\
\text { 2) } E_{I}^{\prime} \neq 0\end{array}$ & $\begin{array}{c}\mathcal{O}\left(\varepsilon^{2} h+h^{2}\right) \\
\mathcal{O}\left(\varepsilon^{2}+h^{2}\right)\end{array}$ & $\begin{array}{c}\mathcal{O}\left(h^{2}+\varepsilon^{2} h^{2}\right) \\
\mathcal{O}\left(\varepsilon^{2} h+h^{2}\right)\end{array}$ & $\begin{array}{c}\mathcal{O}\left(\varepsilon^{2} h+h^{2}\right) \\
\mathcal{O}\left(\varepsilon^{2}+h^{2}\right)\end{array}$ & $\begin{array}{c}\mathcal{O}\left(h^{2}+\varepsilon^{2} h^{2}\right) \\
\mathcal{O}\left(\varepsilon^{2} h+h^{2}\right)\end{array}$ & $\begin{array}{c}\mathcal{O}\left(\varepsilon^{2} h+h^{2}\right) \\
\mathcal{O}\left(\varepsilon^{2}+h^{2}\right)\end{array}$ & $\begin{array}{c}\mathcal{O}\left(h^{2}+\varepsilon^{2} h^{2}\right) \\
\mathcal{O}\left(\varepsilon^{2} h+h^{2}\right)\end{array}$ \\
\hline
\end{tabular}

the first analysis, yet not produce evidence in support of the second one. Maybe there are some latent mechanism to explore and more detailed analysis to make in the cylinder case.

\section{Appendix C}

In this appendix we supply some numerical tests for non AP schemes. The schemes inspected are with discrete diffusion coefficient Choice 1 and discrete boundary flux-limiter Bnd1, i.e., Schemes (a-Bnd1), (b-Bnd1) and (c-Bnd1). They are applied to a cylindrical symmetrical problem with nonhomogeneous Neumann boundary condition.

Example C.1. Consider the radiation diffusion system (4.1)-(4.2) on spatial domain $(0,1) \times(\delta, 1)$. For its zero-th order asymptotic equation (4.3), we suppose the boundary and initial conditions are as follows

$$
\begin{aligned}
& E^{(0)}(0, r, t)=\frac{1}{6}\left(\frac{r}{3} e^{-r t}+\frac{\alpha r}{3}+1\right), \\
& E^{(0)}(1, r, t)=\left(e^{-1}+\frac{1}{6}\right)\left(\frac{r}{3} e^{-r t}+\frac{\alpha r}{3}+1\right) ; \\
& \frac{\partial E^{(0)}}{\partial r}(x, \delta, t)=\frac{(1-\delta t) e^{-\delta t}+\alpha}{3}\left(x e^{-x}+\frac{1}{6}\right), \\
& E^{(0)}(x, 1, t)=\left(\frac{1}{3} e^{-t}+\frac{\alpha+3}{3}\right)\left(x e^{-x}+\frac{1}{6}\right) ; \\
& T^{(0)}(x, r, 0)=\left[\frac{(\alpha+1) r}{3}+1\right]^{\frac{1}{4}}\left(x e^{-x}+\frac{1}{6}\right)^{\frac{1}{4}}, \\
& E^{(0)}(x, r, 0)=\left[\frac{(\alpha+1) r}{3}+1\right]\left(x e^{-x}+\frac{1}{6}\right) .
\end{aligned}
$$


For its first order asymptotic equation (4.4), we suppose

$$
\begin{aligned}
& E^{(1)}(0, r, t)=\gamma\left(\frac{1}{3}\right)^{\frac{1}{2}}\left(2 r e^{-r t}+\beta r+\frac{20}{3}\right) \\
& E^{(1)}(1, r, t)=\gamma\left(e^{-1}+\frac{1}{3}\right)^{\frac{1}{2}}\left(2 r e^{-r t}+\beta r+\frac{20}{3}\right) ; \\
& \frac{\partial E^{(1)}}{\partial r}(x, \delta, t)=\gamma\left[2(1-\delta t) e^{-\delta t}+\beta\right]\left(x e^{-x}+\frac{1}{3}\right)^{\frac{1}{2}} \\
& E^{(1)}(x, 1, t)=\gamma\left(2 e^{-t}+\frac{3 \beta+20}{3}\right)\left(x e^{-x}+\frac{1}{3}\right)^{\frac{1}{2}} ; \\
& T^{(1)}(x, r, 0)=\gamma\left[\frac{(\beta+2) r}{4}+\frac{5}{3}\right]\left[\frac{(\alpha+1) r}{3}+1\right]^{-\frac{3}{4}}\left(x e^{-x}+\frac{1}{3}\right)^{\frac{1}{2}}\left(x e^{-x}+\frac{1}{6}\right)^{-\frac{3}{4}} \\
& E^{(1)}(x, r, 0)=\gamma\left[(\beta+2) r+\frac{20}{3}\right]\left(x e^{-x}+\frac{1}{3}\right)^{\frac{1}{2}}
\end{aligned}
$$

Then we take $T^{(0)}+\varepsilon T^{(1)}$ and $E^{(0)}+\varepsilon E^{(1)}$ as the initial and boundary conditions of the system.

With $\alpha=-1, \beta=-2, \gamma=1$ and $\delta=0$ in (C.1) and (C.2), it represents the cylindrical symmetrical problem with homogeneous Neumann boundary condition in Example 5.7, for which the good behavior of the AP schemes with Bnd 2 has been observed in Section 5 .

In the following, by taking $\alpha=150, \beta=2, \gamma=400$ and $\delta=0.0001$, we detect the three non-AP schemes on a system without natural boundary condition.

Fig. 8 plots the maximum zero-th and first order discrete asymptotic errors of the schemes at $t=0.1$ on a $32 \times 32$ mesh. Although the zero-th order discrete asymptotic errors for energy seem linearly convergent, those for temperature do not. Moreover, the
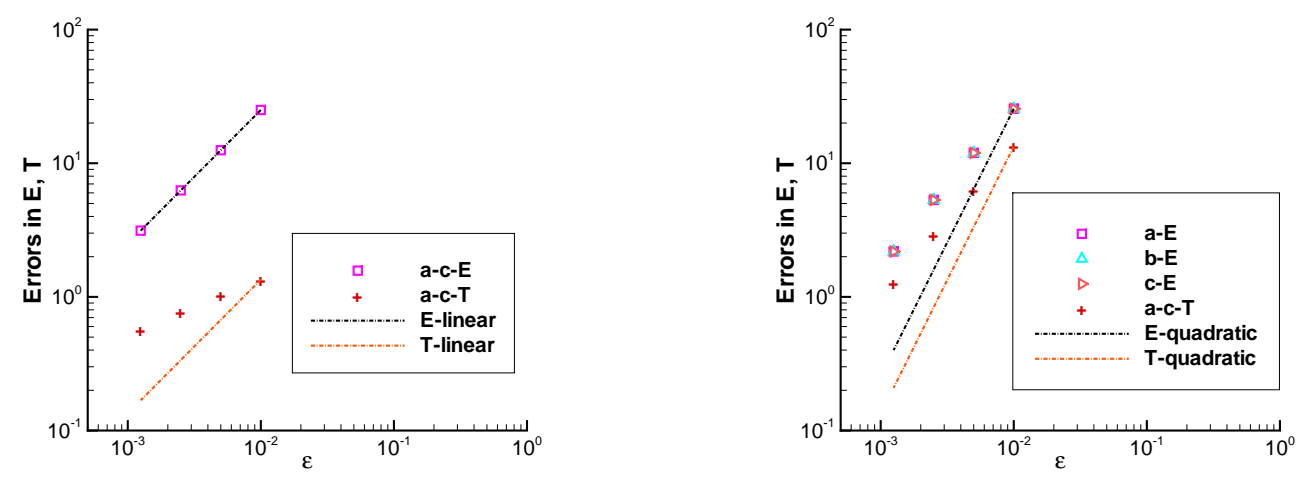

Figure 8: The zero-th (left) and first (right) order discrete asymptotic errors for Example C.1. 

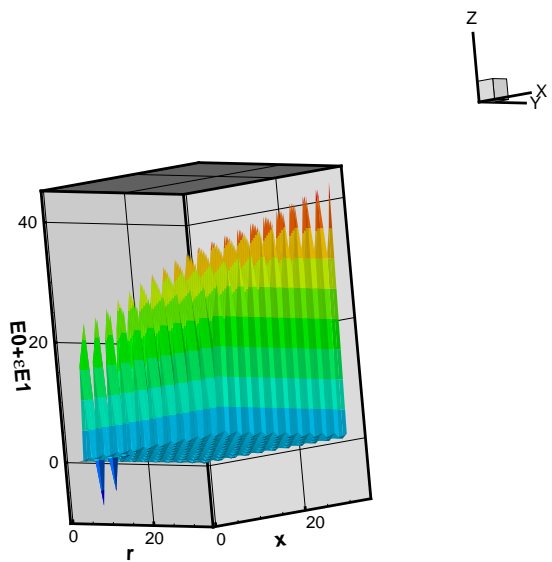

Figure 9: The first order discrete "asymptotic" solution for Example C.1.

first order errors for neither energy nor temperature are second order convergent.

Fig. 9 illustrates the corresponding first order discrete "asymptotic" solution $E^{(0)}+$ $\varepsilon E^{(1)}$ with $\varepsilon=1 / 100$, in which negative numerical energy appears at some points. It demonstrates the schemes are not well-behaved asymptotic-preserving.

\section{References}

[1] F. Filbet and S. Jin, A class of asymptotic-preserving schemes for kinetic equations and related problems with stiff sources, J. Comput. Phys., 229 (2010) 7625-7648.

[2] P. Degond, Asymptotic-preserving schemes for fluid models of plasmas, Panor. Synth., 39-40 (2013) 1-90.

[3] D. Knoll, J. Morel, L. Margolin and M. Shashkov, Physically motivated discretization methods, a strategy for increased predictiveness, Los Alamos Sci., 29 (2005) 188-212.

[4] C. Buet, B. Després and E. Franck, Design of asymptotic preserving finite volume schemes for the hyperbolic heat equation on unstructured meshes, Numer. Math., 122 (2012) 227-278.

[5] B. L. Guo and Y. Q. Han, Diffusion limit of small mean free path of transfer equation in R3, Transp. Theory Stat. Phys., 40 (2011) 243-281.

[6] M. Frank, A. Klar, E. W. Larsen and S. Yasuda, Time-dependent simplified PN approximation to the equations of radiative transfer, J. Comput. Phys., 226 (2007) 2289-2305.

[7] G. Bal and Y. Maday, Coupling of transport and diffusion models in linear transport theory, ESAIM:M2AN, 36 (2002) 69-86.

[8] J. Jang, F. Y. Li, J. M. Qiu and T. Xiong, High order asymptotic preserving DG-IMEX schemes for discrete-velocity kinetic equations in a diffusive scaling, J. Comput. Phys., 281 (2015) 199-224.

[9] E. W. Larsen and G. C. Pomraning, Asymptotic analysis of nonlinear Marshak waves, SIAM J. Appl. Math., 19 (1980) 201-212.

[10] R. M. Rauenzahn, V. A. Mousseau and D. A. Knoll, Temporal accuracy of the nonequilibrium radiation diffusion equations employing a Saha ionization model, Comput. Phys. Commun., 172 (2005) 109-118. 
[11] D. A. Knoll, W. J. Rider and G. L. Olson, Nonlinear convergence, accuracy, and time step control in nonequilibrium radiation diffusion, J. Quant. Spectrosc. Radiat. Transf., 70 (2001) 25-36.

[12] P. N. Brown, D. E. Shumaker and C. S. Woodward, Fully implicit solution of large-scale non-equilibrium radiation diffusion with high order time integration, J. Comput. Phys., 204 (2005) 760-783.

[13] J. Y. Yue and G. W. Yuan, Picard-Newton iterative method with time step control for multimaterial non-equilibrium radiation diffusion problem, Commun. Comput. Phys., 10 (2011) 844-866.

[14] R. P. Zhang, X. J. Yu, J. Zhu, A. F. D. Loula and X. Cui, Weighted interior penalty method with semi-implicit integration factor method for non-equilibrium radiation diffusion equation, Commun. Comput. Phys., 14:5 (2013) 1287-1303.

[15] D. A. Knoll, R. B. Lowrie and J. E. Morel, Numerical analysis of time integration errors for nonequilibrium radiation diffusion, J. Comput. Phys., 226 (2007) 1332-1347.

[16] X. Cui, G. W. Yuan and Z. J. Shen, Asymptotic analysis of discrete schemes for nonequilibrium radiation diffusion, J. Comput. Phys., 313 (2016) 415-429.

[17] C. E. Siewert and J. R. Thomas Jr., Radiative transfer calculations in spheres and cylinders, J. Quant. Spectrosc. Radiat. Transf., 34:1 (1985) 59-64.

[18] A. I. Shestakov, M. K. Prasad, J. L. Milovich, N. A. Gentile, J. F. Painter and G. Furnish, The radiation-hydrodynamic ICF3D code, Comput. Methods Appl. Mech. Engrg., 187 (2000) 181-200.

[19] P. G. Martin, C. Rogers and G. B. Rybicki, Half-range moment methods for radiative transfer in spherical geometry. II - Implementation of the method, Astrophys. J., 284 (1984) 317-326.

[20] J. Chen and C.-W. Shu, Improvement on spherical symmetry in two-dimensional cylindrical coordinates for a class of control volume Lagrangian schemes, Commun. Comput. Phys., 11:4 (2012) 1144-1168.

[21] J. P. Apruzese, J. L. Giuliani and S. B. Hansen, Benchmarking multilevel, 2-D cylindrical radiation transport in a high energy density plasma environment, High Energ. Dens. Phys., 8:3 (2012) 231-237.

[22] C. D. Sijoy and S. Chaturvedi, TRHD: Three-temperature radiation-hydrodynamics code with an implicit non-equilibrium radiation transport using a cell-centered monotonic finite volume scheme on unstructured-grid, Comput. Phys. Commun., 190 (2015) 98-119.

[23] R. G. McClarrenv and R. B. Lowrie, Manufactured solutions for the P1 radiationhydrodynamics equations, J. Quant. Spectrosc. Radiat. Transf., 109 (2008) 2590-2602.

[24] J. S. Warsa and J. D. Densmore, Manufactured solutions in the thick diffusion limit, Nucl. Sci. Eng., 166 (2010) 36-47.

[25] B. Su and G. L. Olson, Non-grey benchmark results for two temperature non-equilibrium radiative transfer, J. Quant. Spectrosc. Radiat. Transf., 62 (1999) 279-302.

[26] K. Ghosh, Analytical benchmark for non-equilibrium radiation diffusion in finite size systems, Ann. Nucl. Energy, 63 (2014) 59-68.

[27] L. I. Sedov, Similarity and Dimensional Methods in Mechanics, New York: Academic Press, 1959.

[28] G. L. Olson, Efficient solution of multi-dimensional flux-limited nonequilibrium radiation diffusion coupled to material conduction with second-order time discretization, J. Comput. Phys., 226 (2007) 1181-1195.

[29] N. N. Yanenko, The Method of Fractional Steps, The Solution of Problems of Mathematical Physics in Several Variables, Springer, 1971. 